\title{
Parsec-scale properties of brightest cluster galaxies
}

\author{
E. Liuzzo ${ }^{1,2}$, G. Giovannini ${ }^{1,2}$, M. Giroletti ${ }^{1}$, and G. B. Taylor ${ }^{3,4}$ \\ 1 INAF Istituto di Radioastronomia, via Gobetti 101, 40129 Bologna, Italy \\ e-mail: liuzzo@ira.inaf.it \\ 2 Dipartimento di Astronomia, Università di Bologna, via Ranzani 1, 40127 Bologna, Italy \\ 3 Department of Physics and Astronomy, University of New Mexico, Albuquerque NM 87131, USA \\ 4 also Adjunct Astronomer at the National Radio Astronomy Observatory, USA
}

Received 16 December 2009 / Accepted 1 February 2010

\section{ABSTRACT}

\begin{abstract}
We present new VLBI observations at $5 \mathrm{GHz}$ of a complete sample of brightest cluster galaxies (BCGs) in nearby Abell clusters (distance class <3). Combined with data from the literature, we provide parsec-scale information for 34 BCGs. Our analysis of the parsec-scale radio emission of BCGs and the cluster X-ray properties finds a possible dichotomy between BCGs in cool-core clusters and those in non-cool-core clusters. Among resolved sources, those in cool-core clusters tend to have two-sided parsec-scale jets, while those in less relaxed clusters have predominantly one-sided parsec-scale jets. We suggest that this difference is caused by the interplay between the jets and the surrounding medium. The one-sided structure in non-cool-core clusters may be due to Doppler boosting effects in relativistic, intrinsically symmetric jets whereas two-sided morphology in cool core clusters is probably related to the presence of heavy and mildly relativistic jets that have been decelerated on the parsec-scale. Evidence of recurrent activity is also found in BCGs in cool-core clusters.
\end{abstract}

Key words. galaxies: clusters: general - galaxies: nuclei - galaxies: jets - galaxies: structure - radio continuum: galaxies

\section{Introduction}

Brightest cluster galaxies (BCGs) are a unique class of objects (Lin \& Mohr 2004). These galaxies are the most luminous and massive galaxies in the Universe, being up to ten times brighter than typical elliptical galaxies, and having large characteristic radii (tens of kiloparsecs, Schombert 1986). Most BCGs are cD galaxies with extended envelopes over hundreds of kiloparsecs, but they can also be giant $\mathrm{E}$ and $\mathrm{D}$ galaxies. They exhibit a small dispersion in luminosity, making them excellent standard candles. We refer e.g. to Hoessel et al. (1980) for a detailed discussion of the absolute magnitude distribution of BCGs, which is not correlated to the dynamic equilibrium of their host clusters (Katayama et al. 2003). The optical morphology often shows evidence of past or recent galaxy mergers, such as multiple nuclei. They tend to lie very close to peaks of the cluster X-ray emission and to have velocities close to the cluster rest frame velocity. All these properties indicate that they might have experienced an unusual formation history compared to other E galaxies. These galaxies are closely related to the collapse and formation of the cluster: recent models suggest that BCGs must form earlier, and that galaxy merging within the cluster, during collapse within a cosmological hierarchy, is a viable scenario for their formation (Bernardi et al. 2006). In many cool core clusters BCGs often have blue excess light indicative of recent star formation with colors that imply starbursts occurring over the past 0.01-1 Gyr (McNamara 2004). Line-emitting nebulae surround approximately a third of all BCGs (Crawford et al. 1999), for example NGC 1275 in Perseus (Conselice et al. 2001) and A1795 (Cowie et al. 1983) exhibiting extended filamentary nebulae (up to $50 \mathrm{kpc}$ from the central galaxy), some of which are cospatial with soft X-ray filaments. Moreover, many of these BCGs also contain reservoirs of $10^{8}-10^{11.5} M_{\odot}$ of molecular hydrogen (Edge 2001).

In the radio band, it is well established that BCGs have peculiar properties (Burns et al. 1981). They are more likely to be radio-loud than other galaxies of the same mass (Best et al. 2006; Dunn et al. 2010). Their radio morphology often also shows evidence of a strong interaction with the surrounding medium: some BCGs have a wide angle tail structure (WAT) that is either extended on the kiloparsec-scale (e.g., 3C 465 in A2634, Sakelliou \& Merrifield 1999), or of small size (e.g. NGC 4874 in Coma cluster, Feretti \& Giovannini 1985); others are diffuse and amorphous sources, either extended (3C 84 in Perseus, Pedlar et al. 1990) or of small size (e.g., the BCG in A154, Feretti \& Giovannini 1994). These last two sources are rare in the general radio population, but frequently present in BCGs and in particular in BCGs located in cooling core clusters of galaxies.

Radio-loud AGNs in BCGs have been proposed as a potential solution to the cooling-flow problem (McNamara et al. 2005). As a consequence of radiative cooling, the temperature in the central regions of the cluster is expected to drop. However, XMM-Newton and Chandra observations of cooling core clusters have shown that the temperature of cluster cores is $~ 30 \%$ higher than expected and also the amount of cooling gas is only about 10\% of that predicted (Peterson et al. 2002). X-ray cavities in the emitting gas coincident with radio lobes demonstrate the interplay between the radio activity of BCGs and the slowing or arrest of cooling in cluster centers (Bîrzan et al. 2008; Dunn et al. 2006). Very deep Chandra observations of the Perseus and Virgo clusters (Fabian et al. 2003, 2006) detected approximately spherical pressure waves in these clusters. These "ripples" are excited by the expanding radio bubbles and the dissipation of their energy can provide a quasi-continuous heating of the X-ray emitting gas (Ruszkowski et al. 2004). 
Despite these results, many important properties of BCGs are poorly known. Not all BCGs are strong radio sources and cyclic activity with a moderate duty cycle is necessary to justify the deceleration of the cooling processes in clusters where the $\mathrm{BCG}$ is not a high power radio source. Moreover, it is unclear if radio properties of BCGs in cooling flow clusters systematically differe from those of BCGs in merging clusters. We note that in cooling clusters the kiloparsec-scale morphology of BCGs is often classified as a mini-halo (Gitti et al. 2007; Govoni et al. 2009), but extended "normal" sources are also present (e.g., Hydra A; Taylor 1996), while in merging clusters the most common morphology is a WAT source, but point-like and core-halo sources are also present.

On the parsec-scale, BCGs have been poorly studied as a class, data being available only for the mostly famous, bright radio galaxies. In some of these cases, these BCGs resemble to normal FRI radio galaxies with relativistic collimated jets. Parsec-scale jets are usually one-sided because of Doppler boosting effects (e.g., 3C 465 in A2634 and 0836+29 in A690, Venturi et al. 1995), although there are also cases where two-sided symmetric jets are detected in VLBI images, and the presence of highly relativistic jets is uncertain (e.g. 3C 338 in A2199, Gentile et al. 2007, and Hydra A in A780, Taylor 1996).

To investigate the BCG properties on the parsec-scale, we selected a sample of BCGs unbiased with respect to their radio and X-ray properties. We present the sample in Sect. 2; in Sect. 3 we provide information about the observations and the data reduction; in Sect. 4 we provide notes about individual sources; in Sect. 5 we describe the statistical properties of our complete sample; in Sect. 6 we present general BCG properties including data from the literature, and finally, in Sects. 7 and 8 we provide the discussion and the conclusion, respectively.

We used the following set of cosmological parameters: $\Omega_{\mathrm{m}}=$ $0.3, \Omega_{\lambda}=0.7, H_{0}=70 \mathrm{~km} \mathrm{~s}^{-1} \mathrm{Mpc}^{-1}$. We define the spectral index $\alpha$ to be $S \propto v^{-\alpha}$, where $S$ is the flux density at frequency $v$.

\section{A complete BCG sample}

To search for links between the parsec-scale properties of BCGs in rich clusters of galaxies and the cluster properties, we need to identify a sample of clusters with no selection effects in terms of $\mathrm{X}$-ray and radio properties, and at limited distance for obvious sensitivity and angular resolution reasons.

We therefore defined an unbiased complete sample by selecting all BCGs in nearby (distance class lower than 3) Abell clusters with a Declination greater than $0^{\circ}$. All clusters have been included with no selection on the cluster conditions (e.g., cooling) and no selection on the BCG radio power. The Abell catalog is the most complete and well studied sample of rich clusters, and our declination limit (larger than $0^{\circ}$ ) will not affect our results. This sample is not redshift limited but is representative of all nearby $(z<0.04)$ clusters of galaxies.

We present the sample in Table 1. For sources detected by our new observations the nuclear position is derived from our image with an estimated uncertainty $\leq 0.1$ mas. For undetected sources ((xn) and sources discussed in the literature), we give in Table 1 the available coordinates (see notes in the Table 1 for the references).

Our complete cluster sample is composed of the BCGs of 23 Abell clusters. The nearest cluster is A262 with redshift = $0.0161(\mathrm{DC}=1)$ and the more distant is A1213 with $z=0.0468$ $(\mathrm{DC}=2)$. Some BCGs such as 3C 84 and 3C 465 are well known and studied on parsec-scale with publicly available data, and we did not obtain new data for them. In Table 1, in the last column, we indicate these sources with (!). We used (*) for binary clusters: where two well defined substructures are present. In these clusters, we give the name of each BCG for the respective sub-clusters. This is the case for A1314, A1367 and A2151. We note also (in the table (**)) that the BCG of A400 is a dumbbell radiosource with two optical nuclei, 3C 75A and 3C 75B. We analyzed the radio emission associated with both. In this way, the BCGs that composed our complete sample are comprised of 27 objects.

\section{Observations and data reduction}

To complete our knowledge of the parsec-scale structure of sources in our sample, we performed new VLBA observations at $5 \mathrm{GHz}$ for 23 sources. Observations of 10 BCGs were carried out in 2007 June, while the remaining 13 targets of the complete sample were observed between 2008 February and March (see Table 2).

All data were obtained in phase referencing mode. Each source was observed for $\sim 3 \mathrm{~h}$. The data were correlated in Socorro, NM, USA. Postcorrelation processing used the NRAO AIPS package and the Caltech Difmap packages. We followed the same scheme for the data reduction of all data sets. We first applied ionospheric and Earth orientation parameters (EOP) corrections. By executing VLBACALA, we then corrected for sampler offsets and applied the amplitude calibration. With VLBAPANG, we corrected the antenna parallactic angles and with VLBAMPCL we removed the instrumental delay residuals. All calibrator data were globally fringe fitted. After flagging bad data, we obtain accurate models for the calibrators, which we used to improve the amplitude and phase calibration for the entire data set. Final maps were obtained with DIFMAP by averaging over IFs and in time. After one step of editing to obtain a good clean model of the source, we applied multi-iterations of self-calibration in phase to sources with a correlated flux density $>5 \mathrm{mJy}$ and eventually amplitude self-calibration to sources with signal-to-noise ratio $>5$.

In Table 2, we report the image parameters for our final maps. Typically, the error in flux density resulting from the calibration is $\sim 6 \%$. On average, the resolution of the final 2007 data maps is better $(3 \times 1.5$ mas $)$ to that of 2008 images $(4 \times 2$ mas $)$ because of the great number of antennas (10 instead of 9) available in the observations of 2007 than in 2008 (Saint Croix VLBA antenna was not present). The noise level is $\sim 0.1 \mathrm{mJy} / \mathrm{beam}$, and the detection rate of our new VLBA observations is $12 / 23$ $(\sim 52 \%)$. For detected sources, the nuclear source position has been estimated with an error $\leq 1$ mas. We have to note that the detection rate is likely influenced by the fact that some BCGs could have an unreliable position derived from arcsecond images. For these sources, we imaged VLBI fields as large as a few arcsecs with no time or/and frequency average allowing us to recover in some cases the nuclear source. More discussion about the detection rate of our BCG sample is given in Sect. 5.

\section{Notes on individual sources}

Here we provide a brief description of all the BCGs in our complete sample, together with some information about the large scale structure and cluster conditions. We present contour images for the parsec-scale radio emission for all resolved sources in our new VLBA observations.

NGC 708 (B2 0149+35) in Abell 262. This cluster is one of the most conspicuous condensations in the Pisces-Perseus 
Table 1. The complete BCG sample.

\begin{tabular}{|c|c|c|c|c|c|c|c|c|}
\hline Abell Cl. & $\mathrm{DC}$ & $z$ & $\mathrm{kpc} / \operatorname{arcsec}$ & $\mathrm{BCG}$ & $\mathrm{RA}_{J 2000}$ & $\operatorname{Dec}_{J 2000}$ & $H P B W$ & Notes \\
\hline 262 & 0 & 0.0161 & 0.328 & NGC 708 & 015246.458 & 360906.494 & $0.003^{\prime \prime}, v$ & \\
\hline 347 & 1 & 0.0187 & 0.380 & NGC 910 & 022526.77 & 414927.3 & $45^{\prime \prime}, x 1^{\prime}$ & \\
\hline \multirow[t]{2}{*}{400} & 1 & 0.0232 & 0.468 & $3 \mathrm{C} 75 \mathrm{~A}$ & 025741.650 & 060120.717 & $0.0065^{\prime \prime}, v$ & ** \\
\hline & & & & $3 \mathrm{C} 75 \mathrm{~B}$ & 025741.563 & 060136.873 & $0.006^{\prime \prime}$, & ** \\
\hline 407 & 2 & 0.0470 & 0.922 & UGC 2489 & 030151.813 & 355019.587 & $0.005^{\prime \prime}, v$ & \\
\hline 426 & 0 & 0.0176 & 0.358 & $3 \mathrm{C} 84$ & 031948.160 & 413042.104 & $0.0018^{\prime \prime}, ! 1$ & \\
\hline 539 & 2 & 0.0205 & 0.415 & UGC 3274 & 051638.91 & 062526.3 & $45^{\prime \prime}, x 1$ & \\
\hline 569 & 1 & 0.0196 & 0.397 & NGC2 329 & 070908.006 & 483655.736 & $0.0035^{\prime \prime}, v$ & \\
\hline 576 & 2 & 0.0381 & 0.755 & CGCG261-059 & 072206.980 & 555230.566 & $0.004^{\prime \prime}, v$ & \\
\hline 779 & 1 & 0.0226 & 0.457 & NGC 2832 & 091946.87 & 334459.6 & $45^{\prime \prime}, x 1$ & \\
\hline 1185 & 2 & 0.0304 & 0.608 & NGC 3550 & 111038.50 & 284604.0 & $5^{\prime \prime}, x 3$ & \\
\hline 1213 & 2 & 0.0468 & 0.918 & $4 C 29.41$ & 111634.619 & 291517.120 & $0.003^{\prime \prime}, v$ & \\
\hline 1228 & 1 & 0.0350 & 0.697 & IC 2738 & 112123.06 & 342124.0 & $5^{\prime \prime}, x 3$ & \\
\hline \multirow[t]{2}{*}{1314} & 1 & 0.0341 & 0.679 & IC 708 & 113359.222 & 490343.428 & $0.0022^{\prime \prime}, v$ & * \\
\hline & & & & IC 712 & 113449.313 & +490440.54 & $5^{\prime \prime x 3}$ & * \\
\hline \multirow[t]{2}{*}{1367} & 1 & 0.0215 & 0.435 & NGC 3842 & 114402.161 & 195659.03 & $5^{\prime \prime}, x 5$ & * \\
\hline & & & & $3 \mathrm{C} 264$ & 114505.014 & 193622.94 & $0.0035^{\prime \prime} ! 2$ & * \\
\hline 1656 & 1 & 0.0232 & 0.468 & NGC 4874 & 125935.796 & 275733.259 & $0.0027^{\prime \prime}, v$ & \\
\hline 2147 & 1 & 0.0356 & 0.708 & UGC 10143 & 160217.027 & 155828.30 & $5^{\prime \prime}, x 3$ & \\
\hline \multirow[t]{2}{*}{2151} & 1 & 0.0371 & 0.737 & NGC 6041 & 160435.794 & 174317.551 & $0.0032^{\prime \prime}, v$ & * \\
\hline & & & & NGC 6047 & 160508.897 & +174354.31 & $5^{\prime \prime}, x^{3}$ & * \\
\hline 2152 & 1 & 0.0374 & 0.742 & UGC 10187 & 160527.176 & 16268.4 & $5^{\prime \prime}, x^{3}$ & \\
\hline 2162 & 1 & 0.0320 & 0.639 & NGC 6086 & 161235.59 & 292904.8 & $5^{\prime \prime}, x 5$ & \\
\hline 2197 & 1 & 0.0303 & 0.606 & NGC 6173 & 162944.887 & 404841.880 & $0.004^{\prime \prime}, v$ & \\
\hline 2199 & 1 & 0.0303 & 0.606 & 3 C 338 & 162838.267 & 393304.15 & $0.0014^{\prime \prime}, ! 3$ & \\
\hline 2634 & 1 & 0.0312 & 0.624 & $3 \mathrm{C} 465$ & 233558.97 & 264516.18 & $0.0025^{\prime \prime}, ! 4$ & \\
\hline 2666 & 1 & 0.0265 & 0.533 & NGC 7768 & 235058.549 & 270850.416 & $0.0035^{\prime \prime}, v$ & \\
\hline
\end{tabular}

Notes. In Col. 1 we report the name of the Abell cluster, in Col. 2 the distance class (DC), in Col. 3 the redshift $z$ of the cluster, in Col. 4 we report the kpc/arcsec conversion for each cluster, in Col. 5 the name of the BCG, in Cols. 6 and 7 we give the RA and Dec in J2000 coordinates of the BCG. In Col. 8 we give the angular resolution of maps used to estimate the core coordinates. We use ${ }^{(v)}$ for the detected objects whose coordinates are taken by the VLBA maps and ${ }^{(! n)}$ for well known radiosources with public VLBI data: ${ }^{(! 1)}$ from Taylor et al. (2006a), (!2) from Lara et al. (1997); Baum et al. (1997); Lara et al. (1999), ('3) from Gentile et al. (2007) and ${ }^{(14)}$ from Venturi et al. (1995). We use ${ }^{(x n)}$ for undetected radiosources for which coordinates are taken from: ${ }^{(x 1)}$, from NVSS ; ${ }^{(x 2)}$ from NED; ${ }^{(x 3)}$ from FIRST; ${ }^{(x 4)}$ from radio data of Feretti \& Giovannini $(1994)$; ${ }_{(x 5)}$ from Ledlow \& Owen (1995). In the notes: ${ }^{(*)}$ is for binary clusters for which we give the name of BCGs of the two sub-clusters; ${ }^{(* *)}$ indicates a dumbbell radiosource for which we consider the radioemission from both optical nuclei.

supercluster. Its redshift is 0.0161. It is a spiral-rich cluster, characterized by the presence of a central X-ray source centered on a D/cD galaxy. Braine \& Dupraz (1994) detect molecular gas and suggest that it contains a cooling flow that may contribute to the central X-ray emission. Observations show a double gaseous component.

The BCG has a dust lane almost perpendicular to the direction of its radio emission (Ebneter \& Balick 1985), which extends along the direction PA $=70^{\circ}$ (Parma et al. 1986). Blanton et al. (2004) discuss the interaction between the cooling gas and the radio source, which shows at arcsecond resolution a core with a symmetric structure. The total $1.4 \mathrm{GHz}$ flux density is $78 \mathrm{mJy}$ (Blanton et al. 2004). This gives a power at $1.4 \mathrm{GHz}$ of $P_{1.4 \mathrm{GHz}}=4.7 \times 10^{22} \mathrm{~W} / \mathrm{Hz}$, classifying the source as a fairly weak, double-lobed FR I galaxy (Fanaroff \& Riley 1974).

On parsec-scale, we observed an unresolved structure with a total flux density of $3.2 \mathrm{mJy}$ - about $64 \%$ of the arcsecond core flux density. The lack of visible jets and the low core radio power are in agreement with the suggestion that the core is in a low activity phase and that the offset of the radiative cooling is due to several outburst episodes (Clarke et al. 2009).

NGC 910 in Abell 347. This cluster at $z=0.0187$ has been classified as a small cooling cluster by White et al. (1997). No powerful radio galaxy is seen in its central region. The BCG shows in NVSS (NRAO VLA Sky Survey) images a slightly resolved and faint emission with a flux density of about $2 \mathrm{mJy}$, which corresponds to a total power $P_{1.4 \mathrm{GHz}}=1.6 \times 10^{21} \mathrm{~W} / \mathrm{Hz}$.

Our VLBA observations did not detect a radio source to a $5 \sigma$ limit of $<0.5 \mathrm{mJy} /$ beam.

3C 75 in Abell 400. This cluster has a redshift $z=0.0235$. The central, elliptical-rich population is composed of two subgroups separated by $700 \mathrm{~km} \mathrm{~s}^{-1}$. These groups are not apparent in the galaxy distribution projected on the sky. From this, we can conclude that a merger is taking place, close to the line-of-sight. Abell 400 does not have a cooling core (Eilek \& Owen 2002). The central BCG galaxy is an interesting case of a dumbbell galaxy, with two components separated by $\sim 7.2 \mathrm{kpc}$ in projection and $453 \mathrm{~km} \mathrm{~s}^{-1}$ in velocity. Twin radio jets depart from each of the two optical nuclei 3C 75A and 3C 75B (Owen et al. 1985). The two radio sources have similar total radio power at $1.4 \mathrm{GHz}$ ( $\log P_{1.4} \sim 24.3$ Parma et al. 1991), but different nuclear flux density. The brightest core is $3 \mathrm{C} 75 \mathrm{~B}$ in the north with a core flux density at $1.4 \mathrm{GHz}$ of $S_{\text {core, } 1.4} \sim 37.6 \mathrm{mJy}$ compared to $10.5 \mathrm{mJy}$ for 3C 75A. The large scale radio structure is classified as FRI (Morganti et al. 1993). The jets are strongly bent by the cluster gas, and on large scales they intertwine and merge (Fig. 1). The strong asymmetry of the source to the north and east might suggest motion of both nuclei to the southwest relative to the cluster gas. We observed both nuclei, 3C 75A and 3C 75B at mas resolution (see Fig. 1). On the parsec-scale, both radio sources are 
A\&A 516, A1 (2010)

Table 2. Image parameters for naturally weighting maps of new VLBA $5 \mathrm{GHz}$ data.

\begin{tabular}{|c|c|c|c|c|c|c|}
\hline $\begin{array}{c}\text { Epoch } \\
\text { yy-mm-dd }\end{array}$ & BCG & Calibrator & $\begin{array}{c}H P B W \\
\text { mas } \times \text { mas, deg }\end{array}$ & $\begin{array}{c}\text { noise } \\
\text { mJy/beam }\end{array}$ & $\begin{array}{c}\text { Peak } \\
\text { mJy/beam }\end{array}$ & $\begin{array}{l}\text { Total flux density } \\
\text { mJy }\end{array}$ \\
\hline \multirow[t]{2}{*}{$2007-06-08$} & NGC 2329 & $\mathrm{~J} 0712+5033$ & $3.31 \times 1.66,30.20$ & 0.10 & 62.4 & 77.1 \\
\hline & NGC 708 & J0156+3914 & $2.92 \times 1.57,-15.90$ & 0.11 & 3.2 & 3.2 \\
\hline \multirow[t]{4}{*}{ 2007-06-14 } & IC 708 & $\mathrm{~J} 1153+4931$ & $2.24 \times 1.81,-4.25$ & 0.10 & 35.5 & 44.2 \\
\hline & IC 712 & $\mathrm{~J} 1153+4931$ & $2.23 \times 1.8,-6.94$ & 0.09 & - & - \\
\hline & NGC 3842 & $\mathrm{~J} 1125+2005$ & $3.05 \times 1.81,2.99$ & 0.14 & - & - \\
\hline & NGC 4874 & $\mathrm{~J} 1257+3229$ & $2.73 \times 1.83,-7.18$ & 0.10 & 7.37 & 10.1 \\
\hline \multirow[t]{4}{*}{ 2007-06-17 } & NGC 6041 & $\mathrm{~J} 1606+1814$ & $3.20 \times 1.70,-6.47$ & 0.09 & 7.63 & 7.83 \\
\hline & NGC 6047 & $\mathrm{~J} 1606+1814$ & $3.27 \times 1.68,-8.09$ & 0.11 & - & - \\
\hline & UGC 10143 & $\mathrm{~J} 1606+1814$ & $3.28 \times 1.70,6.67$ & 0.12 & - & - \\
\hline & UGC 10187 & $\mathrm{~J} 1606+1814$ & $3.31 \times 1.7,-7.57$ & 0.11 & - & - \\
\hline \multirow[t]{3}{*}{ 2008-02-08 } & NGC 910 & $\mathrm{~J} 0219+4727$ & $3.73 \times 2.23,29.50$ & 0.10 & - & - \\
\hline & $3 \mathrm{C} 75 \mathrm{~A}$ & $\mathrm{~J} 0249+0619$ & $6.40 \times 2.21,25.4$ & 0.13 & 20.2 & 22.0 \\
\hline & $3 \mathrm{C} 75 \mathrm{~B}$ & $\mathrm{~J} 0249+0619$ & $5.80 \times 4.31,19.7$ & 0.13 & 37.6 & 44.5 \\
\hline 2008-02-09 & UGC 2489 & $\mathrm{~J} 0310+3814$ & $4.87 \times 2.23,16.80$ & 0.11 & 2.6 & 3.5 \\
\hline \multirow{2}{*}{$2008-02-16$} & NGC 7768 & $\mathrm{~J} 2347+2719$ & $3.51 \times 1.40,-7.15$ & 0.09 & 0.72 & 1.3 \\
\hline & UGC 3274 & $\mathrm{~J} 0517+0648$ & $3.51 \times 1.53,0.28$ & 0.08 & - & - \\
\hline 2008-02-17 & NGC 2832 & J0919+3324 & $3.63 \times 1.68,23.10$ & 0.10 & - & - \\
\hline $2008-02-22$ & $4 C 29.41$ & $\mathrm{~J} 1103+3014$ & $3.04 \times 1.63,-4.56$ & 0.07 & 36.7 & 39.2 \\
\hline \multirow[t]{2}{*}{$2008-02-28$} & NGC 3550 & $\mathrm{~J} 1103+3014$ & $3.02 \times 1.62,-6.37$ & 0.09 & - & - \\
\hline & IC 2738 & $\mathrm{~J} 1127+3620$ & $2.73 \times 1.69,-17.20$ & 0.13 & - & - \\
\hline \multirow[t]{3}{*}{$2008-03-16$} & CGCG 261-059 & $\mathrm{J} 0742+5444$ & $4.03 \times 1.63,-32.30$ & 0.11 & 1.9 & 3.2 \\
\hline & NGC 6173 & $\mathrm{~J} 1652+3902$ & $4.07 \times 1.94,-3.03$ & 0.11 & 1.1 & 1.3 \\
\hline & NGC 6086 & $\mathrm{~J} 1634+3203$ & $4.78 \times 2.20,-2.54$ & 0.11 & - & - \\
\hline
\end{tabular}

Notes. For each source (Col. 2), we give the epoch of observation (Col. 1), the calibrator (Col. 3), the beam size and PA (Col. 4), the noise (Col. 5), the intensity peak (Col. 6), and the total flux density (Col. 7) of final naturally weighted maps.

one sided with the main jet aligned with the kiloparsec structure. In agreement with the kpc scale behavior, $3 \mathrm{C} 75 \mathrm{~A}$ is fainter than 3C 75B. The nucleus of 3C 75A has a flux density $S \sim 19.9 \mathrm{mJy}$ and the jet has $S \sim 2.14 \mathrm{mJy}$ with an extension of $\sim 4$ mas. The core of $3 \mathrm{C} 75 \mathrm{~B}$ has $S \sim 37.6 \mathrm{mJy}$ and the jet has $\sim 7 \mathrm{mJy}$ with an extension of $\sim 14$ mas. The high nuclear flux density of 3C 75A in the VLBI image with respect to the arcsecond core flux density at $1.4 \mathrm{GHz}$ suggests a self-absorbed structure and a possible source variability.

UGC 2489 in A407. No evidence for a cooling core has been found for this cluster. The BCG appears to be embedded in a diffuse optical halo within a region $\sim 60 \mathrm{kpc}$ in size (Crawford et al. 1999). In radio images of arcsecond resolution, it shows a complex extended structure with two lobes and tails oriented east-west. The largest angular size is $\sim 250$ arcsec and the total flux density at $20 \mathrm{~cm}$ is $S_{\mathrm{t}, 20} \sim 728 \mathrm{mJy}$. The core has $S_{\mathrm{c}, 20} \leq$ $10 \mathrm{mJy}$, the east lobe+tail is $\sim 110$ arcsec extended with $S_{\mathrm{E}, 20} \sim$ $305 \mathrm{mJy}$, and the west one is both brighter $\left(S_{\mathrm{W}, 20} \sim 416 \mathrm{mJy}\right)$ and larger ( $\sim 23$ arcsec) (Bondi et al. 1993).

On a mas scale (Fig. 2), the source appears one-sided with a core flux density $\sim 2.6 \mathrm{mJy}$. The jet direction is NW-SE with an extension of 6 mas and flux density $\sim 0.9 \mathrm{mJy}$.

3C 84 in A426 The Perseus cluster, A426, is the most X-ray luminous cluster in the nearby universe, and the prototypical cooling core cluster. Shocks and ripples are clearly evident in the deep Chandra image of Perseus (Fabian et al. 2005, 2006), and could provide steady heating of the cluster center (Fabian et al. 2006). In Perseus, the AGN manifests itself directly as a bright radio source known as Perseus A or 3C 84, associated with the early-type cD galaxy NGC 1275 . As one of the brightest compact radio sources in the sky, 3C 84 has been studied in some detail (Vermeulen et al. 1994; Taylor \& Vermeulen 1996; Silver et al. 1998; Walker et al. 2000). The radio source 3C 84 is well known to contain jets which have been studied on a variety of scales (Pedlar et al. 1990; Dhawan et al. 1998; Silver et al. 1998; Walker et al. 2000). Taylor et al. (2006a) detect linear polarization from the bright jet component $\mathrm{S} 1$ in $3 \mathrm{C} 84$ at 5, 8, 15, and $22 \mathrm{GHz}$ at a level of 0.8 to $7.5 \%$ that increases with frequency. Furthermore, there is some suggestion at $8.4 \mathrm{GHz}$ and above that the polarization is extended. The detection of core polarization is less than $0.1 \%$ for all frequencies except for $22 \mathrm{GHz}$ for which it is less than $0.2 \%$.

The radio morphology is quite complex (Asada et al. 2009; Lister 2001; Agudo et al. 2007) exhibiting a core with two opposite radio-jets, the southern jet consisting of components moving down a diffuse jet and finally expanding into an amorphous component at 12 mas. Krichbaum et al. (1992) showed that the inner jet components move at $0.1 \mathrm{c}$ and that after a major bend the jet speed accelerates.

The jet morphology has been imaged on a variety of scales. At high frequency (and resolution), we compared the VLBA image at $43 \mathrm{GHz}$ (Lister 2001) with the $86 \mathrm{GHz}$ image by Lee et al. (2008). Despite different epochs and resolutions, we tentatively identify the core with component "E" in Lister (2001), which is the only self-absorbed structure (inverted spectrum) between 43 and $86 \mathrm{GHz}$. The source therefore appears initially one-sided, and later becomes two-sided.

At lower frequency and resolution, there is a clear equivalence between the parsec-scale structure and jets on a kiloparsec scale (Pedlar et al. 1990). Looking through VLBA images from $22 \mathrm{GHz}$ to $5 \mathrm{GHz}$, the two-sided structure starts out appearing more symmetric and then at lower frequencies it becomes asymmetric. Walker et al. (1998, 2000) explained this as the effects of free-free absorption of the counter-jet from the surrounding torus. VLBA data at $15 \mathrm{GHz}$ (Lister et al. 2009) measure proper motion in the jet and counterjet and estimate $\gamma=0.6$ and $\theta=11^{\circ}$. In VLBA data at $5 \mathrm{GHz}$ (Taylor et al. 2006a), the largest source angular size is $\sim 35$ mas $(\sim 12 \mathrm{pc})$, the core flux density is 

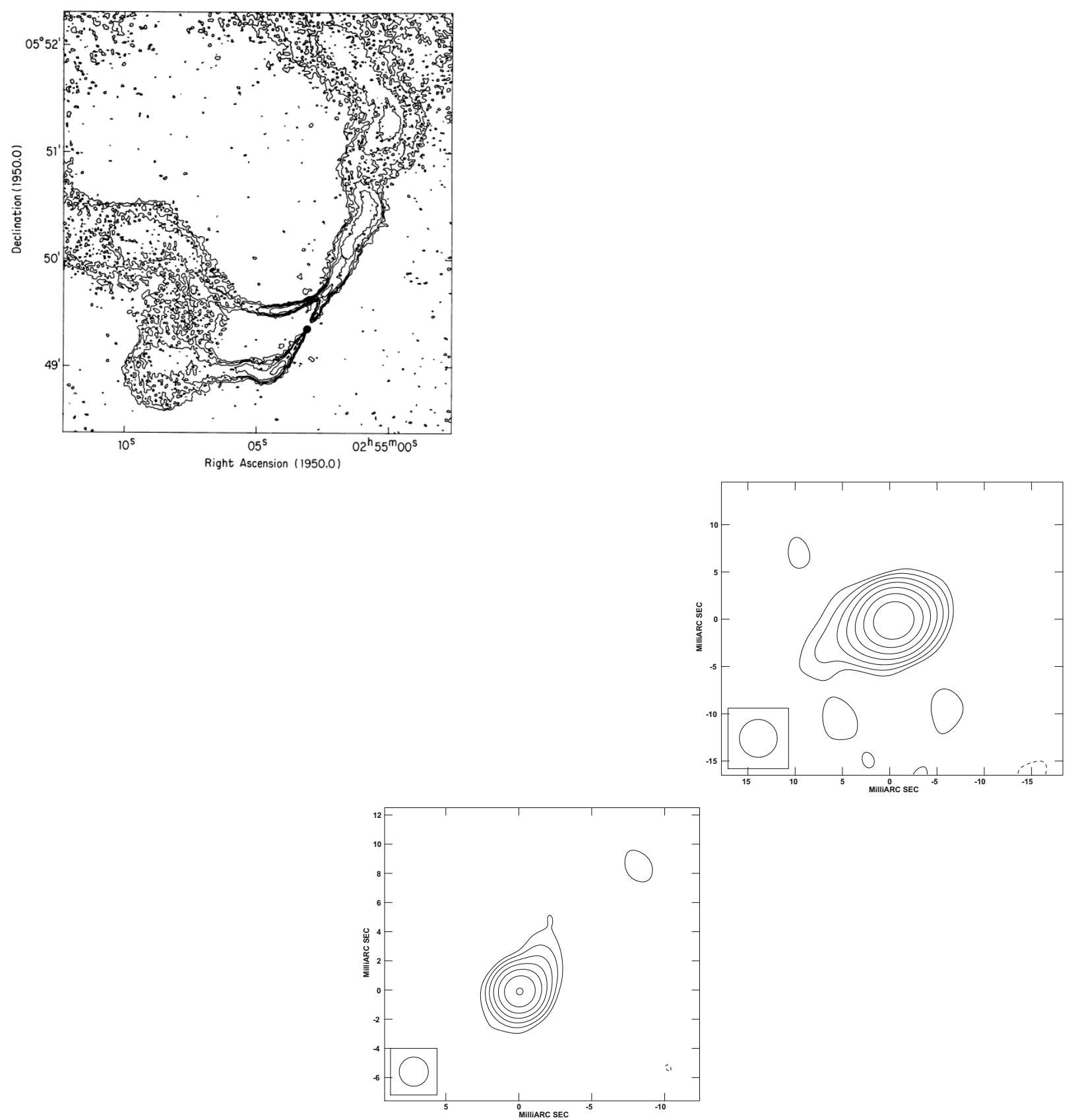

Fig. 1. The double BCG in the non cool core cluster A400. Left: VLA image at $5 \mathrm{GHz}$ of 3C 75A and 3C 75B from Owen et al. (1985). Contour intervals are $(-1,1,2,4,8.16,32,64,128,256,512) \times 0.1$ mJy per clean beam. Center: one-sided $5 \mathrm{GHz}$ VLBA tappered image of 3C 75A. Contour levels are $-0.3,0.3,0.6,1.2,2.4,4.8,9.6$ and $19.2 \mathrm{mJy} /$ beam. The peak flux density is $19.9 \mathrm{mJy} / \mathrm{beam}$, the noise level is $0.1 \mathrm{mJy} / \mathrm{beam}$ and the restoring beam is $2 \times 2 \mathrm{mas}^{2}, \mathrm{PA}=0^{\circ}$. Right: one-sided $5 \mathrm{GHz}$ VLBA tappered image of 3C 75B. Contour levels are $-0.3,0.3,0.6,1.2$, 2.4, 4.8, 9.6 and $19.2 \mathrm{mJy} / \mathrm{beam}$. The peak flux density is $37.2 \mathrm{mJy} / \mathrm{beam}$, the noise level is $0.1 \mathrm{mJy} / \mathrm{beam}$ and the restoring beam is $4 \times 4 \mathrm{mas}{ }^{2}$, $\mathrm{PA}=0^{\circ}$.

$\sim 3.1 \mathrm{Jy}\left(P \sim 2.1 \times 10^{24} \mathrm{~W} / \mathrm{Hz}\right)$, and the total flux density is $23.3 \mathrm{Jy}\left(P \sim 1.6 \times 10^{25} \mathrm{~W} / \mathrm{Hz}\right)$.

UGC 3274 in A539. This cluster is at $z=0.0205$; its internal structure is not clearly understood. Girardi et al. (1997) found that it consists of two systems, both very extended, separated in velocity by over $4000 \mathrm{~km} \mathrm{~s}^{-1}$, but spatially overlapping. The BCG in the NVSS image is not detected $(\leq 1.4 \mathrm{mJy})$, which corresponds to an upper limit to the total power of $\log P \leq$ $21.1(\mathrm{~W} / \mathrm{Hz})$. Our VLBA observations did not detect a radio source to a $5 \sigma$ limit of $<0.4 \mathrm{mJy} /$ beam.

NGC 2329 in Abell 569. No cooling core has been found in this cluster. The galaxy has an exceptionally blue V-I ( 1.20) color (Verdoes Kleijn et al. 1999). The nucleus is clearly bluer than its surroundings. The nuclear emission is slightly resolved and shows a small extension roughly in the direction of the dust protuberance. The galaxy has a small inclined central dust disk.

NGC 2329 has been associated with a Wide-Angle Tail (WAT) radio source with two tails extended for about 4 arcmin. The total power at $1.4 \mathrm{GHz}$ is $\log P=23.48 \mathrm{~W} / \mathrm{Hz}$ and the core power at $5 \mathrm{GHz}$ is $\log P=22.52 \mathrm{~W} / \mathrm{Hz}$ (Feretti \& Giovannini 1985). The extended radio morphology suggests merging.

The VLBA observations at $1.6 \mathrm{GHz}$ of $\mathrm{Xu}$ et al. (2000) detect a one-sided morphology with a peak flux 


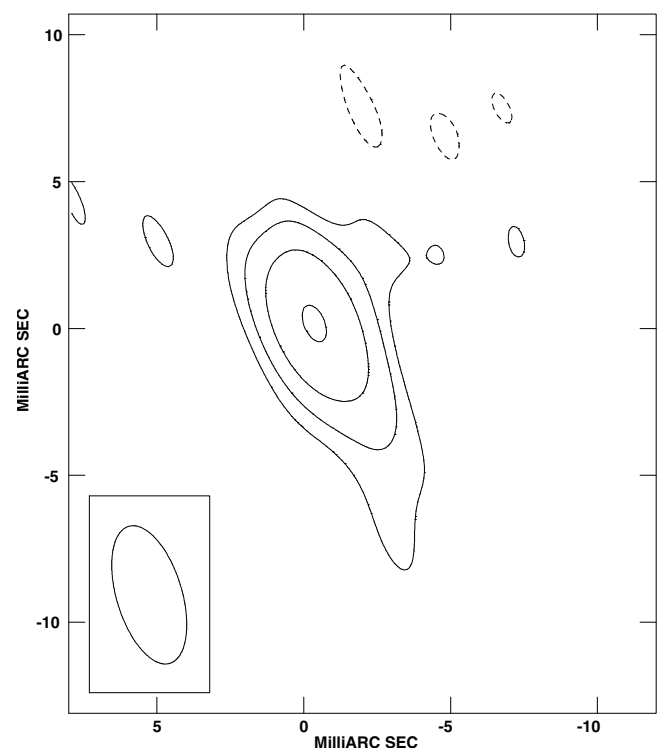

Fig. 2. The $5 \mathrm{GHz}$ VLBA naturally weighted image of UGC 2489, BCG of A407. Contour levels are $-0.31,0.31,0.62,1.24$ and $2.48 \mathrm{mJy} / \mathrm{beam}$. The peak flux density is $2.6 \mathrm{mJy} /$ beam, the noise level is $0.1 \mathrm{mJy} / \mathrm{beam}$ and the restoring beam is $4.87 \times 2.23 \mathrm{mas}^{2}, \mathrm{PA}=16.8^{\circ}$.

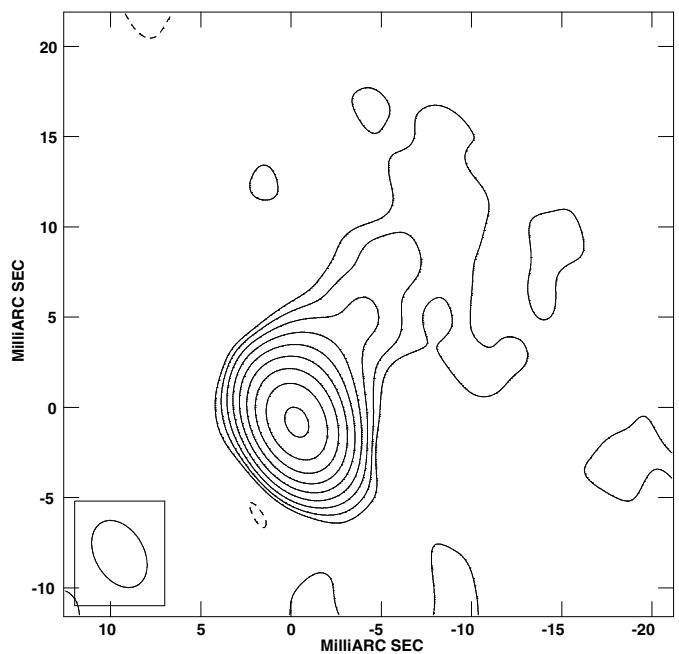

Fig. 3. $5 \mathrm{GHz}$ VLBA tappered map of NGC 2329, BCG of A569.

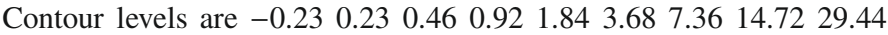
and $58.88 \mathrm{mJy} / \mathrm{beam}$. The peak flux density is $66.8 \mathrm{mJy} / \mathrm{beam}$, the noise level is $0.08 \mathrm{mJy} / \mathrm{beam}$ and the restoring beam is $4.0 \times 2.7 \mathrm{mas}^{2}$, $\mathrm{PA}=30^{\circ}$.

density $\sim 49.7 \mathrm{mJy} /$ beam and total flux density $\sim 59.1 \mathrm{mJy}$. The core-jet is elongated in the direction corresponding to the northeastern radio tail. We confirm this morphology in our data. In our images (Fig. 3), the core has $S \sim 66.8 \mathrm{mJy}$, and the jet has $S \sim 10.3$ mJy with an extension of $\sim 15$ mas. The total spectral index derived from 1.6 and $5 \mathrm{GHz}$ data is $\alpha \sim-0.2$.

CGCG 261-059 in A576: this cluster is not relaxed. The rise in velocity dispersion of the emission-line population towards the cluster core indicates that this population is dynamically aware of the cluster and is probably falling into the cluster for the first time. The observations seem to favor a model in which the core of A576 contains the remnants of a lower mass subcluster (Mohr et al. 1996). The BCG is slightly extended in the NVSS image and exhibits mJy level emission. In the FIRST (Faint Images of the Radio Sky at Twenty-Centimeters) image, it

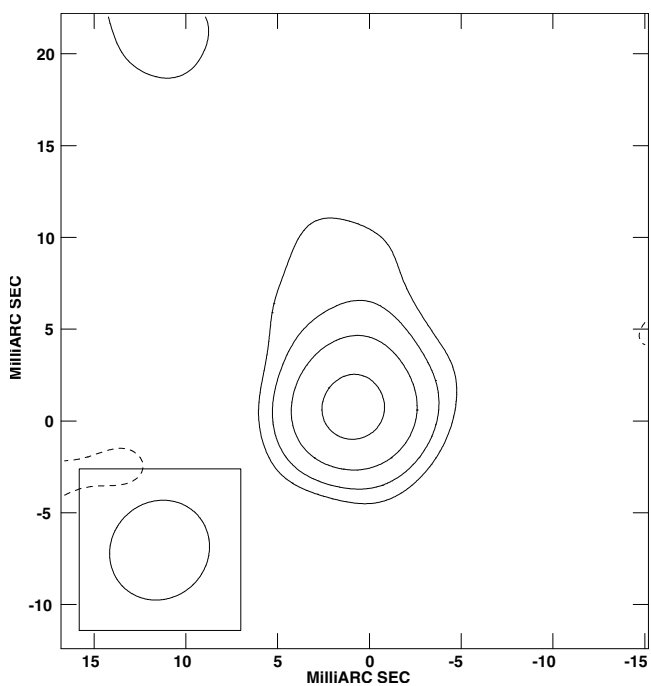

Fig. 4. The $5 \mathrm{GHz}$ VLBA tappered map of CGCG261-059, BCG of A576. Contour levels are $-0.3,0.3,0.6,1.2$ and $2.4 \mathrm{mJy} / \mathrm{beam}$. The peak flux density is $3.0 \mathrm{mJy} / \mathrm{beam}$, the noise level is $0.1 \mathrm{mJy} / \mathrm{beam}$ and the restoring beam is $5.63 \times 5.24 \mathrm{mas}^{2}, \mathrm{PA}=-45.19^{\circ}$.

appears pointlike with a flux density $\sim 3.33 \mathrm{mJy}$ that corresponds to total power $\log P=22.04(\mathrm{~W} / \mathrm{Hz})$.

In our parsec-scale map (Fig. 4), the source is one sided with a core flux density of $1.87 \mathrm{mJy}$. The jet has a flux density of $1.37 \mathrm{mJy}$, and extends for $7 \mathrm{mas}$ in $\mathrm{PA} \sim-20^{\circ}$.

NGC 2832 in Abell 779. This is a poor cluster with no evidence of a cooling core from X-ray data (White et al. 1997). In high-resolution ROSAT HRI soft X-ray observations, NGC 2832 exhibits a strong central emission peak, surrounded by diffuse emission around it. It does not have an extended $\mathrm{cD}$ envelope (Malumuth \& Kirshner 1981). A nearby satellite galaxy, NGC 2831, appears to be tidally interacting with the BCG, despite a relative velocity difference of $1692 \mathrm{~km} \mathrm{~s}^{-1}$ (Lauer 1988). No optical emission lines are present in NGC 2832, which does not have a cooling core (McNamara 1992).

The radio emission associated with the BCG is at the mJy level, unresolved in FIRST images with a flux density $\sim 2.36 \mathrm{mJy}$, which corresponds to a total power $\log P=$ $21.43(\mathrm{~W} / \mathrm{Hz})$. Our VLBA observations did not detect a radio source to a $5 \sigma$ limit of $<0.5 \mathrm{mJy} / \mathrm{beam}$.

NGC3550 in Abell 1185. This cluster is at $z=0.0304$ with no evidence for a cooling core (White et al. 1997). It is undetected in NVSS and FIRST images. In our image, we did not detect any radio emission $\geq 0.45 \mathrm{mJy} /$ beam $(5 \sigma)$.

4C 29.41 in Abell 1213. This cluster is underluminous in the X-rays, just marginally detected by the Einstein and ROSAT satellites. The X-ray images and optical distribution suggest a non-relaxed structure (Jones \& Forman 1999). On kiloparsec scale, this radio source is part of a dumbell galaxy (Trussoni et al. 1997). It is an example of a double and symmetric radio source with a bright one-sided jet. It is classified as a FRI radiogalaxy with a total power at $1.4 \mathrm{GHz} \log P_{1.4 \mathrm{GHz}, \mathrm{t}} \sim 25.35$ and core flux density at $5 \mathrm{GHz}$ of $S_{5 \mathrm{GHz}}$,core $\sim 41 \mathrm{mJy}$. Its linear size is about $81 \mathrm{kpc}$ (Owen \& Ledlow 1997).

On the mas scale (Fig. 5), it has a one-sided morphology, its jet being aligned with the kiloparsec structure. The core flux density is $\sim 36.24 \mathrm{mJy}$, while the jet has $\sim 2.5 \mathrm{mJy}$ and is visible out to $\sim 12$ mas.

IC 2738 in A1228. This cluster is at $z=0.035$. No cooling core has been detected (White et al. 1997). Its BCG is undetected 


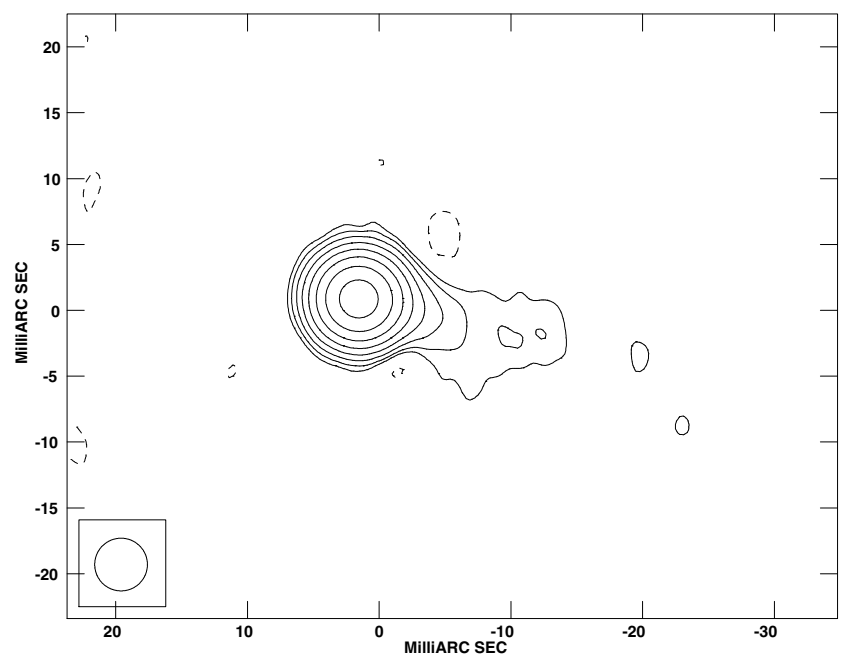

Fig. 5. The $5 \mathrm{GHz}$ VLBA tappered map of 4C29.41, BCG of A1213. Contour levels are $-0.2,0.2,0.4,0.8,1.6,3.2,6.4,12.8$ and $25.6 \mathrm{mJy} / \mathrm{beam}$. The peak flux density is $36.7 \mathrm{mJy} / \mathrm{beam}$, the noise level is $0.07 \mathrm{mJy} / \mathrm{beam}$ and the restoring beam is $4 \times 4 \mathrm{mas}^{2}, \mathrm{PA}=0^{\circ}$.

in NVSS maps with a power $\log P<21.14(\mathrm{~W} / \mathrm{Hz})$. In our image, no detection is present at the $5 \sigma$ level $(0.65 \mathrm{mJy} / \mathrm{beam})$.

IC 712 and IC 708 in Abell 1314. This cluster is a binary cluster with two main condensations visible in the X-ray images. This cluster exhibits very clumpy, elongated X-ray emission, and a strong X-ray centroid shift (Bliton et al. 1998). This elongation is also present in the galaxy distribution, which shows a definite ellipticity, oriented mainly $E-W$ (Flin et al. 1995). The BCG of the main condensation, IC 712, has a WAT structure of very small linear size $(\sim 4.6 \mathrm{kpc})$ with a total flux density at $1.4 \mathrm{GHz}$ of $26.3 \mathrm{mJy}$ (Giovannini et al. 1994). The BCG of the second condensation is IC 708 which also exhibits a WAT structure (Vallee et al. 1979) of greater size ( $\sim 4.5 \operatorname{arcsec})$, total flux density at $1.4 \mathrm{GHZ} S_{1.4 \mathrm{GHz}, \mathrm{t}} \sim 430 \mathrm{mJy}$, and core flux density at $6 \mathrm{~cm}$ of $S_{5 \mathrm{GHz}, \text { core }} \sim 110 \mathrm{mJy}$.

In our maps, IC 712 is undetected at the $5 \sigma$ level (>0.45 mJy/beam). IC 708 (Fig. 6) shows a one-sided structure with a core flux density $S \sim 38.8 \mathrm{mJy}$. The jet is aligned with the kiloparsec structure, and has $S \sim 2.48 \mathrm{mJy}$ and an extension of $\sim 16$ mas from the core.

3C 264 and NGC 3842 in Abell 1367. A 1367 is an X-rayfaint and nearby $(z=0.0215)$ galaxy cluster. It has a secondary peak in its X-ray brightness (Donnelly et al. 1998) located approximately $19^{\prime}$ offset from the primary brightness peak. This cluster is peculiar having extended trails of radio emission behind three irregular galaxies in its periphery (Gavazzi \& Jaffe 1987). It is a complex cluster currently forming at the intersection of two filaments. NCG 3842 and NGC 3862 are its two Brightest Cluster Galaxies.

NGC 3842 is a cD galaxy, which was studied in radio band by Feretti \& Giovannini (1994). From high resolution VLA radio images, NGC 3842 exhibits a small size WAT structure with angular size of $\sim 55$ arcsec and a flux density of $10.5 \mathrm{mJy}$ corresponding to a total power at $1.4 \mathrm{GHz} \log P_{\mathrm{t}, 1.4 \mathrm{GHz}}=$ $21.72 \mathrm{~W} / \mathrm{Hz}$. In our images, it appears to be undetected above $5 \sigma$ ( $>0.7 \mathrm{mJy} /$ beam).

NGC3862 is associated with the strong radio source 3C 264. In VLA images, it has a total power of $\log P=24.97(\mathrm{~W} / \mathrm{Hz})$. On kiloparsec scales, 3C 264 has a head-tailed morphology, prominent core, and a wiggling jet extending toward the northeast that ends in a blob of emission at $28 \operatorname{arcsec}(11.5 \mathrm{kpc})$

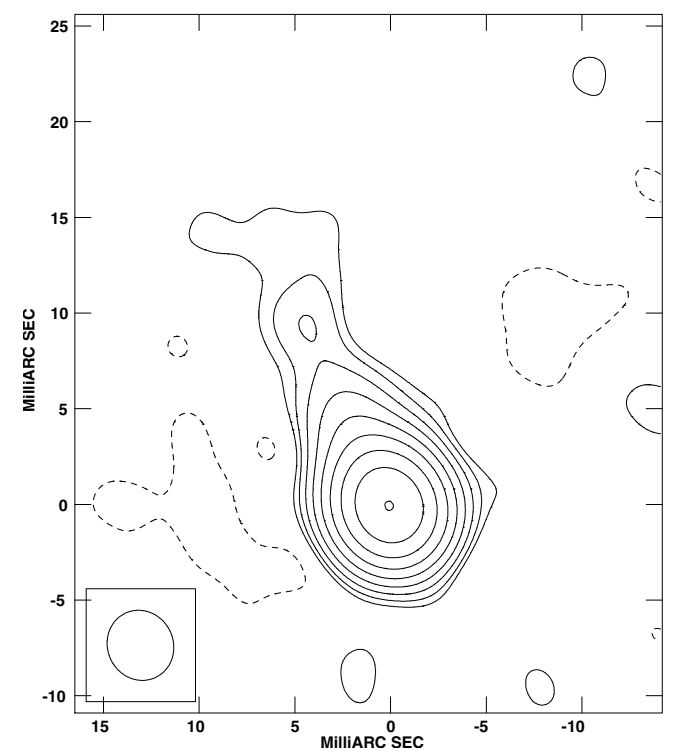

Fig. 6. The $5 \mathrm{GHz}$ VLBA tappered map of IC 708, BCG of the second condensation of the binary cluster A1314. Contour levels are -0.15 , $0.15,0.3,0.6,1.2,2.4,4.8,9.6,19.2$ and $38.4 \mathrm{mJy} /$ beam. The peak flux density is $38.8 \mathrm{mJy} / \mathrm{beam}$, the noise level is $0.05 \mathrm{mJy} / \mathrm{beam}$ and the restoring beam is $3.71 \times 3.43 \mathrm{mas}^{2}, \mathrm{PA}=25.4^{\circ}$.

from the core (Lara et al. 1997). There is evidence of counterjet emission in the southwest direction from the core. Both the jet and counterjet are embedded in a vast and diffuse region of low surface brightness emission, which seems to have been dragged toward the north, possibly revealing the existence of a high density intracluster medium. Simultaneous EVN and MERLIN observations at 5.0 GHz by Lara et al. (1999); Baum et al. (1997) showed for the first time the detailed structure of 3C 264 on sub-kiloparsec scales. It consists of a one-sided jet with evident variations in its morphological properties with distance: i) the strong core and innermost jet $(0-10 \mathrm{pc})$; ii) a wellcollimated and narrow region (10-100 pc); iii) a region with strong widening, kinks, and filaments (100-300 pc); and iv) a faint and narrow region after a jet deflection (300-400 pc from the core). In particular, in the EVN map at $5 \mathrm{GHz}$ (Lara et al. 1999), the source exhibits an unresolved core with a peak flux density $\sim 0.126 \mathrm{Jy} /$ beam and a smooth one-sided jet, extending to 25 mas from the core along $\mathrm{PA} \sim 27^{\circ}$.

NGC 4874 in Abell 1656. This is one of two dominant members of the Coma cluster (Abell 1656) and considered to be the Coma BCG. It is a cD galaxy whit a small size WAT structure. Arcsecond scale properties are discussed in Feretti \& Giovannini (1987), where a possible precessing beam model is discussed. On kiloparsec scales, it has a total flux density at $1.4 \mathrm{GHz}$ of $190 \mathrm{mJy}$ and the core flux density at $6 \mathrm{~cm}$ is $1.1 \mathrm{mJy}$. The radio emission is completely embedded in the optical galaxy. The radio linear size is $7 \mathrm{kpc}$. In the VLA $1.4 \mathrm{GHz}$ image, a gap of radio emission is present between the core and the SW lobe, while a faint jet connecting the core and the NE lobe is detected. At $5 \mathrm{GHz}$, the jets are detected after gaps of $\sim 3$ arcsec on both sides of the core.

In our VLBA image (Fig. 7), the source shows a one-sided structure with a total flux density of $10.1 \mathrm{mJy}$. The core flux density is $7.27 \mathrm{mJy}$. The jet has a flux density $\sim 2.83 \mathrm{mJy}$ and is extended in the direction SW $\left(\mathrm{PA} \sim-15.4^{\circ}\right)$ with an angular size of $\sim 3$ mas.

UGC 10143 in Abell 2147. In the X-ray images, this cluster shows extended elongated emission suggestive of a merger. 


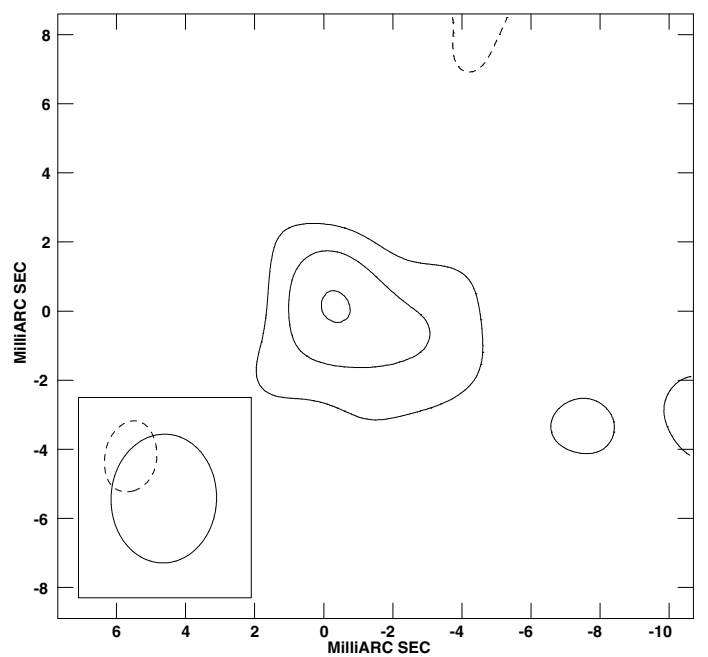

Fig. 7. The $5 \mathrm{GHz}$ VLBA tappered map of NGC 4874, BCG of A1656. Contour levels are -0.240 .240 .48 and $0.76 \mathrm{mJy} / \mathrm{beam}$. The peak flux density is $0.8 \mathrm{mJy} / \mathrm{beam}$, the noise level is $0.08 \mathrm{mJy} / \mathrm{beam}$ and the restoring beam is $3.73 \times 3.05 \mathrm{mas}^{2}, \mathrm{PA}=-2.1^{\circ}$.

(Flin \& Krywult 2006). The BCG is a cD galaxy that has elongated radio emission in the NVSS image. In the high resolution image from FIRST, it shows a WAT morphology of angular size $\sim 20$ arcsec and total flux density $\sim 8.03 \mathrm{mJy}$. On mas scale, we did not detect a radio source, the $5 \sigma$ limit being $<0.6 \mathrm{mJy} / \mathrm{beam}$.

NGC 6041A and NGC 6047 in Abell 2151. This Abell cluster is also known as the Hercules cluster. It is a highly structured cluster despite the apparently regular velocity distribution of the main field. This cluster should be considered as an ongoing cluster merging (Girardi et al. 1997). This cluster exhibits two peaks in the X-ray emission (Bird et al. 1995). There is only marginal evidence of soft excess emission in the brightest of the two peaks (Bonamente et al. 2002).

NGC 6041A is the BCG with the first main condensation, while NGC 6047 is the brightest galaxy of the second condensation. In the NVSS image, NGC 6041A exhibits a WAT structure with angular size $\sim 2.5$ arcmin and total flux density $\sim 11.6 \mathrm{mJy}$. In our VLBA map, it appears unresolved with a total flux density $\sim 7.83 \mathrm{mJy}$.

NGC 6047 is classified as an E/S0 and has an unusual optical morphology indicative of a recent merger that severely disrupted the dynamics of an $\mathrm{HI}$ disk whose gas lost its kinematic coherence (Dickey 1997). On the kiloparsec scale, NGC 6047 exhibits an extended FRI structure with a two-sided jet emission (Feretti \& Giovannini 1988). The northern jet is brighter (flux density at $5 \mathrm{GHz} \sim 13.6 \mathrm{mJy}$ ) and more collimated. The total flux density at $1.4 \mathrm{GHz}$ is $\sim 728 \mathrm{mJy}$ and the core flux density at $5 \mathrm{GHz}$ is $\sim 8.3 \mathrm{mJy}$. In our maps, it appears undetected above $5 \sigma=0.55 \mathrm{mJy} / \mathrm{beam}$.

UGC 10187 in A2152. A2152 is one of the major condensations in the Hercules supercluster together with A2147 and A2151. Its BCG is UGC10187, which is also the brightest of a galaxy pair. In the NVSS, this BCG shows an extended ( $\sim$ arcmin in NVSS) tailed radio structure with total flux density $\sim 60.3 \mathrm{mJy}$. In the FIRST image, a gap of $\sim 5$ arcsec between the two tails is present and the nuclear source is not visible. In our VLBI image, no detection was found with a $5 \sigma$ limit of $0.55 \mathrm{mJy} / \mathrm{beam}$.

NGC 6086 in Abell 2162. This Abell cluster is a low X-ray luminosity cluster (Burns et al. 1994). Its BCG is a bright cD galaxy, which hosts a double-lobed radio source. In the NVSS

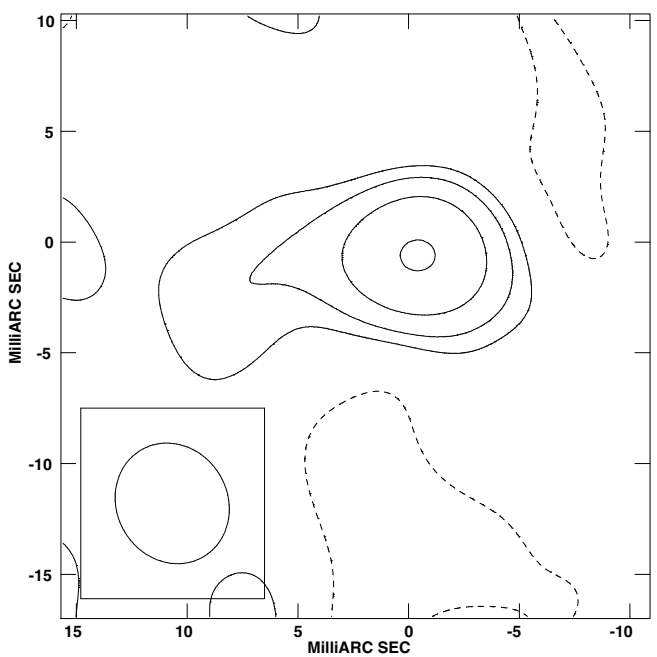

Fig. 8. The $5 \mathrm{GHz}$ VLBA tappered map of NGC 6173, BCG of A2197. Contour levels are -0.190 .190 .380 .76 and $1.52 \mathrm{mJy} / \mathrm{beam}$. The peak flux density is $1.6 \mathrm{mJy} / \mathrm{beam}$, the noise level is $0.06 \mathrm{mJy} / \mathrm{beam}$ and the restoring beam is $5.58 \times 5.0 \mathrm{mas}^{2}, \mathrm{PA}=30^{\circ}$.

image, the total flux density is $\sim 108.7 \mathrm{mJy}$ and its angular size is $\sim 3.5$ arcmin. In the FIRST image, the radio emission is partly resolved out (only $\sim 60 \mathrm{mJy}$ ) and extended over $\sim 2.5 \mathrm{arcmin}$. No clear structure is discernible. The radio spectrum and morphology suggest that it is a relic galaxy where the core radio activity stopped some time ago. Our VLBA observations did not detect a radio source to a $5 \sigma$ limit of $0.55 \mathrm{mJy} /$ beam.

NGC 6173 in A2197. This cluster is one of a few clusters that have an unusual systematic alignment among individual cluster members and is possibly linked to A2199 via supercluster structure to the Hercules region (Gregory \& Thompson 1984).

The BCG shows mJy level emission $(\sim 7 \mathrm{mJy})$ unresolved in FIRST images. On the mas scale, it appears (Fig. 8) one-sided with a total flux density in our VLBA maps of $3.24 \mathrm{mJy}$. In particular, the core has a flux density of $1.87 \mathrm{mJy}$ and the jet is extended to the east with an angular size $\sim 10$ mas and flux density $\sim 1.37 \mathrm{mJy}$.

3 C 338 in Abell 2199. A2199 $(z=0.0304)$ has a prototypical cooling core. A Chandra observation of this cluster has detected a point-like source coinciding with the center of the galaxy NGC6166 (Di Matteo et al. 2001), which is a multiple nuclei $\mathrm{cD}$ galaxy. This galaxy hosts the relatively powerful radio source $3 \mathrm{C} 338$, which emits a total power at $330 \mathrm{MHz}$ of $\log P \sim 25.64 \mathrm{~W} / \mathrm{Hz}$. This radio source has been known for a few decades to have an unusual structure on both large and small scales (Feretti et al. 1993; Giovannini et al. 1998). It is classified as a FR I radio source and shows central optical [O III] line emission (Fisher et al. 1995). On kiloparsec scales 3C 338 has two symmetric extended radio lobes, characterized by a steep spectrum $(\alpha \sim-1.7)$ and misaligned with the central emission. The two radio lobes are connected by a bright filamentary structure. Polarimetric observations by Ge \& Owen (1994) revealed strong rotation measure gradients across most of the extended emission and inferred the presence of cluster magnetic fields. Both the steep radio spectrum and strong filamentary emission may be caused by interactions with the dense intracluster medium (Gentile et al. 2007).

On parsec-scales, 3C 338 has a compact radio core with two short ( $\sim 10 \mathrm{pc})$, symmetric jets. In VLBA maps at $5 \mathrm{GHz}$ (Feretti et al. 1993), the parsec-scale structure shows a central dominant feature (the core emission) with flux density $\sim 92 \mathrm{mJy} / \mathrm{beam}$ 


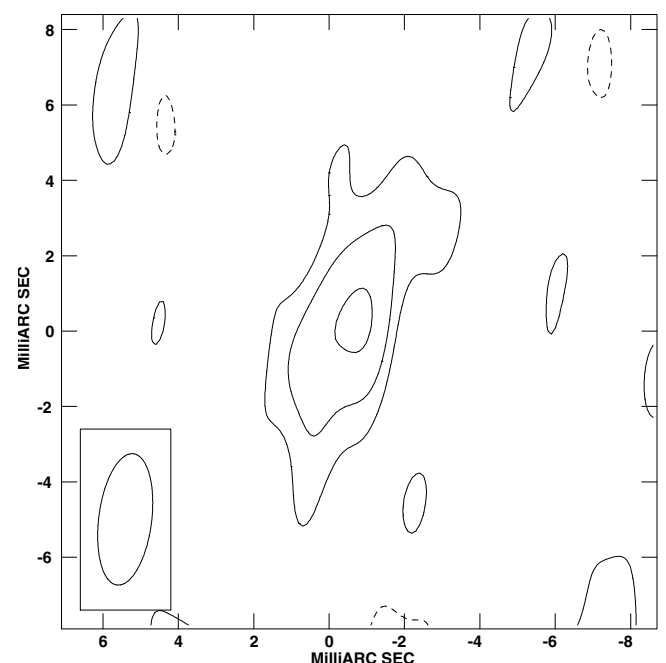

Fig. 9. The $5 \mathrm{GHz}$ VLBA naturally weighting map of NGC 7768, BCG of A2666. Contour levels are -0.170 .170 .34 and $0.64 \mathrm{mJy} / \mathrm{beam}$. The peak flux density is $0.72 \mathrm{mJy} / \mathrm{beam}$, the noise level is $0.09 \mathrm{mJy} / \mathrm{beam}$ and the restoring beam is $3.51 \times 1.40 \mathrm{mas}^{2}, \mathrm{PA}=-7.15^{\circ}$.

and two symmetric jets. The total flux density is $133 \mathrm{mJy}$. The eastern jet shows a couple of low-brightness regions in its center suggesting it could be limb-brightened. The orientation of this structure appears to be very constant in time despite the complex dynamic conditions present in the 3C 338 central regions. Comparing maps obtained at different epochs (Gentile et al. 2007), a change in the parsec-scale morphology is clearly evident and is probably correlated with the arcsecond core flux density variability. The structural changes suggest the presence of proper motion with $\beta \sim 0.4 \mathrm{~h}^{-1}$ on both sides of the core (Gentile et al. 2007). These properties suggest that the extended emission in 3 C 338 is a relic structure that is not related to the present nuclear activity (Giovannini et al. 1998).

3C 465 in Abell 2634. A2634 $(z=0.0322)$ does not show evidence of a cooling core. Its BCG is $3 \mathrm{C} 465$ that is associated with the giant D galaxy NGC 7720. The optical host galaxy exhibits distorted isophotes with two gravitationally bound nuclei surrounded by a common envelope (Venturi et al. 1995). Weak broad $\mathrm{H}_{\alpha}$ and high-ionization emission-lines suggest the presence of faint activity in the optical nucleus. In the radio band, this source has a WAT morphology. Its total power at $408 \mathrm{MHz}$ is $\log P=25.3$. The radio jet, the spots, and the low-brightness tails are strongly polarized. On parsec-scales, the source is onesided with the jet on the same side as the main kiloparsec scale structure. At $5 \mathrm{GHz}$, in VLBA data (Venturi et al. 1995), the core flux density is $168 \mathrm{mJy}$ and the total flux density is $237 \mathrm{mJy}$.

NGC 7768 in Abell 2666. Scodeggio et al. (1995) did not find any evidence of substructures in the central cluster region. Its BCG is a cD galaxy in the center of the cluster. It contains a dusty nuclear disk approximately aligned with the major axis of the galaxy (Grillmair et al. 1994) and radio emission at the mJy level ( $2 \mathrm{mJy}$, which corresponds to $\log P=21.50 \mathrm{~W} / \mathrm{Hz})$.

In our VLBA data (Fig. 9), it shows a one sided structure with a core flux density $S \sim 0.72$ mJy and total flux density $\sim 1.31 \mathrm{mJy}$. The jet is oriented in the direction NW-SE, and has $S \sim 0.59 \mathrm{mJy}$ and an extension of $\sim 4$ mas from the core.

\section{Parsec scale morphology}

Our complete sample consists of 27 BCGs: 23 were observed for the first time with VLBA by ourselves, and for the remaining objects literature radio information on the mas scale are available. The detection rate of our new VLBA observations is $52 \%$ and, adding literature parsec-scale information about the well studied radio sources, the total detection rate at mas resolution of our complete sample is $59 \%(16 / 27)$. In particular, $45 \%(12 / 27)$ are one-sided, 7\%(2/27) have a twosided morphology, 7\%(2/27) show point-like structure, and $41 \%(11 / 27)$ are undetected. Table 3 presents parsec-scale and arcsecond radio properties for all our BCGs (complete sample and extended sample, see Sect. 7).

\subsection{Two-sided morphology}

In our sample, two sources, 3C 84 and 3C 338, show two-sided structure. For both radio sources, the most probable explanation of their properties is that they consist of two oppositely directed, symmetric, and mildly relativistic jets at a modest angle to the line of sight (Walker et al. 1994; Dhawan et al. 1998 for 3C 84 and Giovannini et al. 1998; Gentile et al. 2007 for 3C 338).

Both 3C 84 and 3C 338 are located at the center of cool core clusters. The Perseus cluster, Abell 426, in particular is the most $\mathrm{X}$-ray luminous cluster in the nearby universe, and the prototypical "cooling core" cluster. These two sources show evidence of restarted activity. In 3C 338 there is evidence of restarted radio activity, an extended relic emission and a small scale young structures (Giovannini et al. 1998). Features suggesting the recurrent jet activity of the central engine are also present in $3 \mathrm{C} 84$. At low resolution (frequencies below $5 \mathrm{GHz}$ ), the southern jet extends continuously from the core out to $\sim 100$ mas (Taylor 1996), indicating that $3 C 84$ had previous outbursts. There are also multiple lobe-like structures on the arcminute scale (Pedlar et al. 1990). The synchrotron age of inner lobe (Nagai et al. 2009) and the observed inner proper motions (Asada et al. 2009) are consistent with the inner lobe having been formed by the outburst in the year 1959 (Nesterov et al. 1995).

As discussed in the previous section, 3C 84 appears onesided at $86 \mathrm{GHz}$ in the inner $2.5-3$ mas $(\leq 1 \mathrm{pc})$ and symmetric (two sided) at $\sim 4-5$ mas $(\geq 1.5 \mathrm{pc}$ ) from the core as is evident in the $22 \mathrm{GHz}$ images by Taylor et al. (2006a). The jet/counterjet ratio is $>35$ at $43 \mathrm{GHz}$ at 0.5 mas $(\sim 0.15 \mathrm{pc})$ and $\sim 1$ at 5 mas $(\sim 1.5 \mathrm{pc})$ suggesting a strong jet deceleration. This result cannot be due to free-free absorption effect (Walker et al. 2000), since the one sidedness is visible in the high resolution high frequency image. We note that a similar structure can be detected only here because of the high linear spatial resolution due to the low redshift of 3C 84.

\subsection{One-sided morphology}

In our complete sample, 12 BCGs have one-sided parsec-scale structures. The percentage $(45 \%)$ is in agreement with the percentage of one-sided FRI radio galaxies found in a complete sample of radio galaxies (Liuzzo et al. 2009b): 23/51 (45\%). We note that all 12 BCGs lie in non-cool core clusters.

The values of the jet/counter-jet ratios for the sources of our complete sample are given in Table 3. We used these values together with the core dominance information to estimate the angle and jet velocity (Giovannini et al. 2001). Results are uncertain but consistent with an asymmetry because of Doppler boosting effects.

We note that in all resolved BCG sample sources, the parsecscale jet is aligned with the arcsecond structure: this indicates that no complex strong change in the angular momentum of the 
Table 3. Parameters for BCGs.

\begin{tabular}{|c|c|c|c|c|c|c|c|c|c|c|}
\hline BCG & $\begin{array}{c}\mathrm{pc} \\
\text { structure }\end{array}$ & $\begin{array}{l}\mathrm{j} / \mathrm{cj} \\
\text { ratio }\end{array}$ & $\beta \cos \theta$ & $\begin{array}{l}S_{\mathrm{c}, 5} \\
\mathrm{mJy}\end{array}$ & $\begin{array}{c}\log P_{\mathrm{c}, 5} \\
\mathrm{~W} / \mathrm{Hz}\end{array}$ & $\begin{array}{l}S_{\mathrm{t}, 408} \\
\mathrm{mJy}\end{array}$ & $\begin{array}{c}\log P_{\mathrm{t}, 408} \\
\mathrm{~W} / \mathrm{Hz}\end{array}$ & $\begin{array}{c}S_{\mathrm{VLBI}} / \\
S_{\mathrm{c}, 5 \mathrm{GHz}}(\%)\end{array}$ & $\begin{array}{c}\text { Core } \\
\text { dominance }\end{array}$ & Notes \\
\hline NGC 708 & $\mathrm{c}$ & - & - & 5 & 21.47 & 364 & 23.33 & 60 & 0.26 & 5 \\
\hline NGC 910 & n.d. & - & 2 & - & - & - & - & $<25$ & - & 5 \\
\hline $3 \mathrm{C} 75 \mathrm{~A}$ & $1 \mathrm{~s}$ & $\geq 6.2$ & $\geq 0.35$ & 6 & 21.87 & 190 & 23.37 & 100 & 0.60 & 1 \\
\hline $3 \mathrm{C} 75 \mathrm{~B}$ & $1 \mathrm{~s}$ & $\geq 4.6$ & $\geq 0.3$ & 20 & 22.39 & 190 & 23.37 & 100 & 2 & 1 \\
\hline $3 C 84$ & $2 \mathrm{~s}$ & 2 & 0.14 & $28.17 \times 10^{3}$ & 25.29 & $51.68 \times 10^{3}$ & 25.56 & 80 & 70.79 & 2 \\
\hline UGC 3274 & n.d. & - & - & $<1.4$ & - & - & - & - & - & 5 \\
\hline UGC 2489 & $1 \mathrm{~s}$ & $\geq 2.8$ & $\geq 0.2$ & 4 & 22.32 & $2.83 \times 10^{3}$ & 25.17 & 90 & 0.13 & 3 \\
\hline NGC 2329 & $1 \mathrm{~s}$ & $\geq 4.5$ & $\geq 0.29$ & 160 & 23.14 & 1300 & 24.05 & 50 & 4.3 & 4 \\
\hline CGCG 261-059 & $1 \mathrm{~s}$ & $\geq 1.8$ & $\geq 0.12$ & 1.5 & 21.71 & 6.54 & 22.34 & 100 & 1.8 & 5 \\
\hline NGC 2832 & n.d. & - & - & 2.4 & - & - & - & $<21$ & - & 5 \\
\hline NGC 3550 & n.d. & - & - & $<1.4$ & - & - & - & - & - & 5 \\
\hline 4C 29.41 & $1 \mathrm{~s}$ & $\geq 3.8$ & $\geq 0.26$ & 41 & 23.33 & 135.2 & 23.84 & 100 & 8.71 & 6 \\
\hline IC 2738 & n.d. & - & - & - & - & - & - & - & - & 5 \\
\hline IC 708 & $1 \mathrm{~s}$ & $\geq 6.5$ & $\geq 0.36$ & 110 & 23.47 & 901.1 & 24.39 & 40 & 5.62 & 7 \\
\hline IC 712 & n.d. & - & - & 14.02 & 22.58 & 48.72 & 23.12 & $<3$ & 4.37 & 11 \\
\hline NGC 3842 & n.d. & - & - & 9 & 22.98 & 101.88 & 23.03 & $<8$ & 0.36 & 11 \\
\hline 3C 264 & $1 \mathrm{~s}$ & $\geq 6$ & $\geq 0.34$ & 200 & 23.32 & $17 \times 10^{3}$ & 25.25 & 100 & 1.17 & 8 \\
\hline NGC 4874 & $1 \mathrm{~s}$ & $\geq 1.6$ & $\geq 0.09$ & 1.1 & 21.13 & 351.96 & 23.63 & 100 & 0.08 & 9 \\
\hline UGC 10143 & n.d. & - & - & $<2$ & $<21.77$ & 14.87 & 22.64 & - & $<1.35$ & 5 \\
\hline NGC 6041A & $\mathrm{c}$ & - & - & $\leq 0.9$ & $\leq 21.92$ & 21.5 & 22.84 & 100 & 1.45 & 5 \\
\hline NGC 6047 & n.d. & - & - & 8.3 & 22.42 & 1960 & 24.80 & $<7$ & 0.28 & 12 \\
\hline UGC 10187 & n.d. & - & - & $<2$ & $<21.81$ & 111.7 & 23.56 & - & $<0.41$ & 5 \\
\hline NGC 6086 & n.d. & - & - & $<1$ & $<21.37$ & 201.35 & 23.68 & - & $<0.12$ & 5 \\
\hline NGC 6173 & $1 \mathrm{~s}$ & $\geq 1.4$ & $\geq 0.07$ & 3.7 & 21.89 & 12.97 & 22.44 & 40 & 2.40 & 5 \\
\hline 3C 338 & $2 \mathrm{~s}$ & 2.2 & 0.16 & 480 & 24.01 & $18.12 \times 10^{3}$ & 25.59 & 30 & 3.47 & 10 \\
\hline $3 \mathrm{C} 465$ & $1 \mathrm{~s}$ & $\geq 20$ & $\geq 0.54$ & 246 & 23.74 & $10.38 \times 10^{3}$ & 25.37 & 100 & 2.57 & 8 \\
\hline NGC 7768 & $1 \mathrm{~s}$ & $\geq 1.1$ & $\geq 0.02$ & 0.74 & 21.08 & 2.6 & 21.62 & 100 & 1.17 & 5 \\
\hline B2 0836+29II & $1 \mathrm{~s}$ & $\geq 20$ & $\geq 0.54$ & 131 & 24.31 & 1139 & 25.24 & 100 & 11.20 & 13 \\
\hline Hydra A & $2 \mathrm{~s}$ & 1.2 & 0.04 & 168 & 24.08 & $132 \times 10^{3}$ & 26.98 & 100 & 0.58 & 14 \\
\hline $4 C 26.42$ & $2 \mathrm{~s}$ & 1.4 & 0.07 & 53 & 23.71 & 3153 & 25.48 & 80 & 2.04 & 15 \\
\hline 3C 317 & $2 \mathrm{~s}$ & 1.5 & 0.08 & 310 & 23.93 & $132 \times 10^{3}$ & 26.56 & 100 & 0.72 & 16 \\
\hline B2151+174 & $2 \mathrm{~s}$ & 4 & 0.27 & 164 & 25.40 & 538 & 25.92 & 100 & 53 & 17 \\
\hline PKS 2322-123 & $2 \mathrm{~s}$ & 1.2 & 0.04 & 59.3 & 24.0 & $7.2 \times 10^{3}$ & 26.11 & 80 & 1.66 & 18 \\
\hline PKS 1246-410 & $1 \mathrm{~s}$ & $\geq 9$ & 0.34 & 64.4 & 22.27 & 2463 & 23.86 & 30 & 0.76 & 19 \\
\hline
\end{tabular}

Notes. $S_{\mathrm{t}, 408}$ : 1) From NVSS with $\alpha \sim 0.5$, 2) from Parma et al. (1991) with $\alpha \sim 0.5$; 3) from Pedlar et al. (1990) where $\alpha \sim 0.5$, 4) from Bondi et al. (1993), 5) from Feretti \& Giovannini (1985), 6) from Owen \& Ledlow (1997) with $\alpha \sim 0.5,7$ ) from Vallee et al. (1979) where $\alpha \sim 0.6$, 8) from Feretti \& Giovannini (1994), 9) from Giovannini et al. (2001) and NED information with $\alpha \sim 0.5,10)$ from Feretti \& Giovannini (1987) with $\alpha \sim 0.5,11)$ from Owen \& Ledlow (1997), 12) from Taylor et al. (2002) and NED information, 13) from Venturi et al. (1995), 14) from Taylor et al. (1990); Taylor (1996), 15) from Liuzzo et al. (2009a), 16) from Venturi et al. (2004), 17) from Augusto et al. (2006) and from NED with $\alpha \sim 0.5$ (408 MHz) and $\alpha \sim 0$ (5 GHz), 18) from Taylor et al. (1999) and NED information and 19) from Taylor et al. (2006b) assuming $\alpha=0.5 . S_{\mathrm{c}}$ is for the arcsecond core flux density at $5 \mathrm{GHz}$ and $S_{\mathrm{VLBI}}$ is a correlated flux density at $5 \mathrm{GHz}$ in our VLBI data.

We give the name of the BCG (Col. 1), the parsec-scale morphology (Col. 2: core (c), one sided (1 s) or two sided (2 s) jet structure), the jet/counterjet surface brightness ratio (Col. 3), $\beta \cos \theta$ (Col. 4), the arcsecond core flux density $S_{\mathrm{c}, 5}$ at $5 \mathrm{Ghz}$ (Col. 5), the arcsecond core power $\log P_{\mathrm{c}, 5}$ at $5 \mathrm{GHz}$ (Col. 6), the total arcsecond flux density $S_{\mathrm{t}, 408}(\mathrm{Col} .7)$ and the total arcsecond power $\log P_{\mathrm{t}, 408}(\mathrm{Col} .8)$ at $408 \mathrm{MHz}$, the total VLBI flux density and arcsecond core flux density ratio $S_{\mathrm{VLBI}} / S_{\mathrm{c}, 5}$ at $5 \mathrm{GHz}$ (Col. 9), the core dominance (see Sect. 5.2) (Col. 10). The last column is for the notes.

accreted gas, and no restarted activity with a different inclination of the accretion disk and or central $\mathrm{BH}$ precession occur.

\subsection{Unresolved sources}

At milliarcsecond resolution, two radio sources of our complete sample appear unresolved: NGC 708 in the cooling cluster A262, and NGC 6041A in the merging cluster A2151. for NGC 708, we note that the core dominance, defined as the ratio of the observed to the estimated core radio power according to the relation given in Giovannini et al. (2001) (see also Liuzzo et al. 2009b) is very low (0.25) suggesting that the nuclear activity is in a low phase and for this reason the jets are not visible. NGC 6041A is a faint source $(\log P \sim 22.57(\mathrm{~W} / \mathrm{Hz})$ at $1.4 \mathrm{GHz}$ ) and the parsec-scale jets - if any - are probably too weak to be detected with the present sensitivity.

\subsection{Undetected sources}

In our new VLBA observations, 11 BCGs are undetected above $5 \sigma$. This percentage is high but still significantly lower that in non-BCG ellipticals. The nature of the undetected BCGs is varied and they can be grouped as follows:

- radio quiet sources (5/11): this is the case for UGC 3274 , NGC 2832, NGC 3550, IC 2738, and NGC 910, which do not exhibit any radio emission at arcsecond resolution, 
- radioquiet core (4/11) in a radio galaxy: in these sources, the central AGN was active in the past, but is radio quiet at the time of observations. This is the case: for NGC 6086, a candidate relic radio galaxy; for the WAT NGC 3842 where the arcsecond core is very faint $(\sim 0.26 \mathrm{mJy})$; for UGC 10143 ; and for UGC 10187 where the nuclear source is not detected by the VLA radio images. As expected in these sources the core dominance is low,

- peculiar sources (2/11): NCG 6047 and IC 712. In VLA maps, these sources show radio emission from the core but they appear undetected in our VLBA maps. This may due to an extreme variability of the core emission. Alternatively, these sources could have a complex structure on scales between these allowed by VLBA and VLA with a pc scale low surface brightness that we are unable to map with our VLBA data. In these cases, more sensitive VLBA observations, EVLA observations at high frequencies, or e-Merlin observations will be important to properly study these structures.

\section{Results for the complete sample}

We note that in our complete sample we have 23 clusters of galaxies, and only 5 of them $(22 \%)$ have been defined cooling core clusters. This percentage is lower than values found for example by Hudson et al. (2010), who found that $44 \%$ of galaxy clusters have strong cool cores. However, we note that their statistic is based on X-ray flux limited samples, while our sample has no selection effects related to the X-ray luminosity. If we apply the same constraints of Hudson et al. (2010) to our sample we have only 9 clusters, 3 of them $(33 \%)$ containing a strong cool core, in agreement (note the small numbers) with literature data.

\subsection{Statistical considerations}

- We compare the total flux on VLBA scales with the core arcsecond flux density (Table 3). Over all data, among 16 detected sources, we find that 11/16 (70\%) have a correlated flux density higher than $80 \%$ of the arcsecond flux density. This means that in these sources we imaged most of the mas scale structure and so we can accurately relate the parsec to the kiloparsec structures. In contrast, for 5/16 (30\%), a significant fraction of the arcsecond core flux density is missing in the VLBA images. This suggests variability or the presence of significant structures between $\sim 10$ mas and 1 arcsec that the VLBA may miss because of lack of short baselines. To properly study these structures, future observations with the EVLA at high frequency or with the e-MERLIN array will be necessary.

- We derive the distribution of the total radio luminosities at 1.4 GHz obtained from NVSS (Condon et al. 1998) and FIRST (Becker et al. 1995) and the RLF (Radio Luminosity Function) of the radio loud sources in our complete sample. We compare our sample properties with the results of Best et al. (2007) for their complete sample of BCGs. Because of the lack of spectroscopic information of our BCGs, we are not able to distinguish between radio emission due to star formation and that from to AGN activity. Following Best et al. (2007), we compared only sources with $\log L \geq 22.3$ and we assumed that the detected radio emission of our BCGs is from central AGN.

For our complete sample, we found that the probability of BCGs being radio loud with $\log L \geq 22.3$ is $\sim 87 \%$. This is consistent with the results of Best et al. (2007). We consider this result as evidence that our small sample is representative of the general properties of BCGs.

- Among the sources of our complete sample, there are two, $3 \mathrm{C} 84$ and $3 \mathrm{C} 338$, that exhibit evidence of restarted activity in the radio emission associated with the BCG. Both 3C 84 and 3C 338 lie in Abell clusters that exhibit a cool core. In the case of 3C 84, Nagai et al. (2009) and Agudo et al. (2007) used multifrequency VLBA observations to constrain the timescale of the restarted activity. Agudo et al. (2007) derived a kinematic age, whereas Nagai et al. (2009) estimated the synchrotron age of the radio source. Both authors found an age consistent with a scenario in which the inner $\sim 15$ mas feature is formed by the recent outburst in the year 1959. At low frequencies, the southern jet extends continuously from the core out to $\sim 100$ mas, suggesting that $3 \mathrm{C} 84$ has had multiples outbursts. There are also multiple lobe-like structures on arcminute scales that suggest a recurrent jet activity of the central engine.

For 3C 338, owing to its peculiar morphology and arcsecond core flux variability, Giovannini et al. (1998) suggest that the extended emission is older and unrelated to the present nuclear activity (see also Gentile et al. 2007).

\section{The extended sample}

In term of the X-ray emission of the Abell clusters in our complete sample, clusters can be divided in to coolcore and non cool clusters (Table 4). However, on parsec-scale, we note that those with two-sided structure (3C 84 and 3C 338) are only found in cool core clusters (A 426 and A 2199) and one-sided sources are all observed in non cool core clusters. A comparison between BCGs in cooling and non-cooling clusters shows a difference in their properties of the parsec-scale structures, but numbers are too small to be properly discussed. To improve our statistics, we performed a search of the literature and archive data for VLBI data of BCGs in Abell clusters that have detailed information about the cluster dynamics (X-ray emission) and radio emission at mas and arcsec resolution. To obtain an extended BCG list, we added to our complete sample the following sources:

- B2 0836+29II in A690 (O’Donoghue et al. 1990; Giovannini et al. 2005);

- Hydra A in A780 (Taylor 1996; Wise et al. 2007);

- 4C 26.42 in A1795 (Liuzzo et al. 2009a; Salomé \& Combes 2004);

- 3C317 in A2052 (Venturi et al. 2004);

- B2151+174 in A2390 (Augusto et al. 2006);

- PKS 2322-123 in A2597 (Morris \& Fabian 2005; Taylor et al. 1999):

- PKS 1246-410 (NGC4696) in A3526 (Taylor et al. 2006b).

In Table 4 we provide our results concerning the morphology for the extended sample. We report the Abell cluster of BCG (Col. 1), X-ray cluster properties (Cols. 2 and 3), name of BCG (Col. 4), large scale morphology of BCG (Col. 5), parsec-scale structure (Col. 6) and references (Col. 7). In particular, in Col. 3 we report the values of central mass accretion rate of the cluster derived from the literature (see Col. 7) to provide an idea of the strength of the central gas density.

The extended sample is composed of 34 BCGs: 10 are in cool core clusters and 24 are in non cool core clusters. Table 5 summarizes statistically properties of the complete and the extended sample. 
Table 4. Results for the extended sample.

\begin{tabular}{|c|c|c|c|c|c|c|}
\hline Abell cluster & Cool core & $\begin{array}{c}M_{\text {accr }} \\
M_{\odot} / \mathrm{yr}\end{array}$ & BCG & Large scale & VLBI & Ref. \\
\hline \multirow[t]{2}{*}{ A400 } & $\mathrm{N}$ & $0.0_{-0.0}^{+28.3}$ & $3 \mathrm{C} 75 \mathrm{~A}$ & WAT & one sided & White et al. (1997) \\
\hline & $\mathrm{N}$ & $0.0_{-0.0}^{+28.3}$ & $3 \mathrm{C} 75 \mathrm{~B}$ & WAT & one sided & White et al. (1997) \\
\hline A407 & $\mathrm{N}$ & $4.6_{-4.6}^{+11.8}$ & UGC 2489 & Tail rs & one sided & White et al. (1997) \\
\hline A539 & $\mathrm{N}$ & $2.1_{-2.1}^{+6.8}$ & UGC 3274 & radio quiet & n.d. & White et al. (1997) \\
\hline A569 & $\mathrm{N}$ & $>5.2_{->4.2}^{+0.0}$ & NGC 2329 & WAT & one sided & White et al. (1997) \\
\hline A576 & $\mathrm{N}$ & $17_{-17}^{+47.2}$ & CGCG 261-059 & Tail rs & one sided & White et al. (1997) \\
\hline A690 & $\mathrm{N}$ & $0.0_{-0.0}^{+15.3}$ & B2 0836+29 II & WAT & one sided & Giovannini et al. (2005); White et al. (1997) \\
\hline A779 & $\mathrm{N}$ & $3.1_{-1.1}^{+1.1}$ & NGC 2832 & radio quiet & n.d. & White et al. (1997) \\
\hline A1185 & $\mathrm{N}$ & $0.0_{-0.0}^{+1.1}$ & NGC 3550 & radio quiet & n.d. & White et al. (1997) \\
\hline A1213 & $\mathrm{N}$ & $0.0_{-0.0}^{+11.5}$ & 4C 29.41 & FRI & one sided & White et al. (1997) \\
\hline A1228 & $\mathrm{N}$ & - & IC 2738 & radio quiet & n.d. & - \\
\hline \multirow[t]{2}{*}{ A1314 } & $\mathrm{N}$ & $0.0_{-0.0}^{+3.0}$ & IC 708 & WAT & one sided & White et al. (1997) \\
\hline & $\mathrm{N}$ & $0.0_{-0.0}^{+3.0}$ & IC 712 & small WAT & n.d. & White et al. (1997) \\
\hline \multirow[t]{2}{*}{ A1367 } & $\mathrm{N}$ & $2.3_{-2.3}^{+6.8}$ & NGC 3842 & small WAT & n.d. & White et al. (1997) \\
\hline & $\mathrm{N}$ & $2.3_{-2.3}^{+6.8}$ & 3C 264 & HT & one sided & Lara et al. (1999); White et al. (1997) \\
\hline A1656 & $\mathrm{N}$ & $0.0_{-0.0}^{+1.0}$ & NGC 4874 & small WAT & one sided & White et al. (1997) \\
\hline A2147 & $\mathrm{N}$ & $0.0_{-0.0}^{+14.5}$ & UGC 10143 & small WAT & n.d. & White et al. (1997) \\
\hline \multirow{2}{*}{ A2151 } & $\mathrm{N}$ & $6.3_{-3.2}^{+26.3}$ & NGC 6041 & small WAT & core & White et al. (1997) \\
\hline & $\mathrm{N}$ & $6.3_{-3.2}^{+26.3}$ & NGC 6047 & compact core + symmetric jets & n.d. & White et al. (1997) \\
\hline A 2162 & $\mathrm{~N}$ & - & NGC 6086 & FRI, relic source & n.d. & - \\
\hline A2197 & $\mathrm{N}$ & $2.4_{-2.4}^{+3.0}$ & NGC 6173 & point source & one sided & White et al. (1997) \\
\hline A2634 & $\mathrm{N}$ & $0.0_{-0.0}^{+1.5}$ & 3C 465 & WAT & one sided & Venturi et al. (1995); White et al. (1997) \\
\hline A2666 & $\mathrm{N}$ & $0.0_{-0.0}^{+2.6}$ & NGC 7768 & Tail rs & one sided & White et al. (1997) \\
\hline A3526 & $\mathrm{N}$ & $5.2_{-0.3}^{+0.3}$ & PKS 1246-410 & small tailed rs & one-sided & Taylor et al. (2006b); Hudson et al. (2010) \\
\hline A262 & Y & $9.4_{-4.4}^{+21.2}$ & NGC 708 & double-no core,jets & core & White et al. (1997) \\
\hline A347 & $\mathrm{SCF}$ & $7.8_{-2.7}^{+3.5}$ & NGC 910 & radio quiet & n.d. & White et al. (1997) \\
\hline A426 & Y & $291_{-58}^{+>-7}$ & 3C 84 & Compact core + Halo & two sided & Taylor et al. (2006a); White et al. (1997) \\
\hline A780 & $\mathrm{Y}$ & $222_{-132}^{+98}$ & Hydra A & double & two sided & Taylor (1996); White et al. (1997) \\
\hline A1795 & $\mathrm{Y}$ & $321_{-213}^{+166}$ & $4 C 26.42$ & double & two sided & Liuzzo et al. (2009a); White et al. (1997) \\
\hline A2052 & $\mathrm{Y}$ & $94_{-37}^{+84}$ & 3C 317 & bright core+halo (FRI) & two sided & Venturi et al. (2004); White et al. (1997) \\
\hline A2152 & $\mathrm{Y}$ & $20_{-20}^{+13}$ & UGC 10187 & Tail rs & n.d. & White et al. (1997) \\
\hline A2199 & $\mathrm{Y}$ & $97_{-31}^{+9}$ & 3C 338 & double restarted & two sided & White et al. (1997); Feretti et al. (1993) \\
\hline A2390 & $\mathrm{Y}$ & $247_{-91}^{+43}$ & B2151+174 & MSO & two sided & Augusto et al. (2006); Allen et al. (2001) \\
\hline A2597 & Y & $501_{-512}^{+58}$ & PKS 2322-123 & asymmetric radiosource (FRI) & two sided & Taylor et al. (1999); Chen et al. (2007) \\
\hline
\end{tabular}

Notes. In the first column, we report the Abell cluster of BCG of our sample, in Col. 2, we indicate Y if the Abell cluster shows a cool core, $\mathrm{N}$ if it doesn't, in Col. 3 there are the value of central mass accretion rate of the cluster taken from the literature, in Col. 4 there are the names of BCGs. Column 5 is for the large scale morphology of the BCG: we use WAT for Wide Angle Tail radiosource, HT for head tail radiosource, MSO for medium symmetric source. In Col. 6, we mark the parsec-scale structure: one sided, two sided, core (unresolved) or n.d. for the non detections. In the last column, we give the references for the mas scale structure when the source is yet well studied at mas scale in literature and for values of mass accretion rate given in Col. 3 .

In the expanded sample, we find in cool core clusters:

- $70 \%$ two-sided sources;

- $20 \%$ non detected sources;

- $10 \%$ unresolved structures.

Instead, in non cool core clusters, BCGs show:

- $58 \%$ one-sided morphology;

- $38 \%$ non detected sources;

- $4 \%$ unresolved structures.

\section{Discussion}

The presence of a clear dichotomy between relaxed and non relaxed clusters is evident (Table 5). On mas scale, one-sided structures are only in BCGs in non cool core clusters, two-sided morphologies are only found in BCGs inside cool core clusters.

\subsection{Jet velocities}

We use as a comparison sample the Bologna Complete Sample (BCS) (Giovannini et al. 2001, 2005). This sample is composed of 95 FRI radio galaxies spanning the same radio power range as our BCG sample. It is free of selection effects, in particular in term of jet velocity and orientation. As for our sample, VLBI observations at $5 \mathrm{GHz}$ and kiloparsec morphology information for most of these objects are available and presented in Liuzzo et al. (2009b). Among the results from the BCS study, Giovannini et al. (2005) and Liuzzo et al. (2009b) found that the 
E. Liuzzo et al.: Parsec-scale properties of BCGs

Table 5. BCG counts in the complete (nearby) sample and expanded one.

\begin{tabular}{ccccccc}
\hline \hline Sample & $\begin{array}{c}\text { Cluster } \\
\text { morphology }\end{array}$ & Number & Two-sided & One-sided & Point & N.D. \\
\hline Complete & $\begin{array}{c}\text { cool core } \\
\text { non cool core }\end{array}$ & 5 & $22(40 \%)$ & - & 1 & 2 \\
Expanded & cool core & 10 & $7(70 \%)$ & - & 1 & 9 \\
& non cool core & 24 & - & $14(58 \%)$ & 1 & 9 \\
\hline
\end{tabular}

Notes. We report the number of BCG according to the cluster morphology and parsec-scale morphology. Note that most of the undetected sources in VLBA observations are in BCG that are radio quiet (or faint) in VLA observations (see Sect. 5.3).

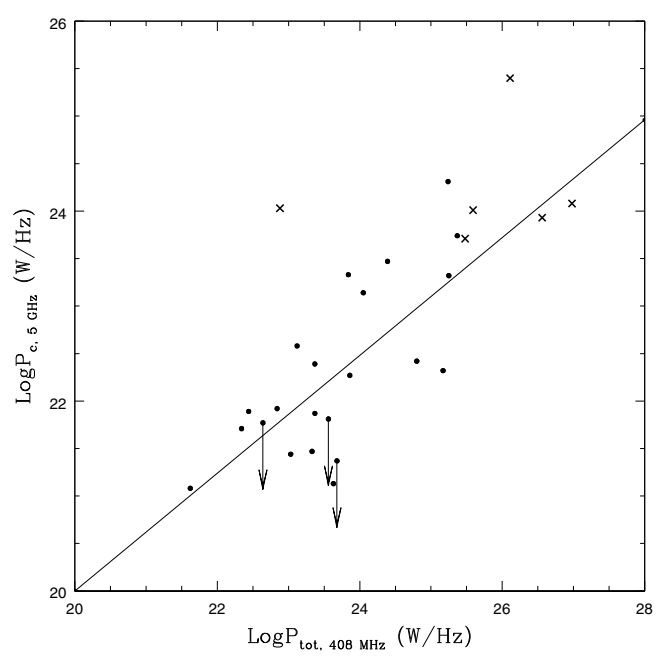

Fig. 10. Total arcsecond radio power at $408 \mathrm{MHz}$ versus observed arcsecond core radio power at $5 \mathrm{GHz}$ for all BCGs of our complete sample with available information. The solid line is the relation found by Giovannini et al. (2001). Crosses (x) represent BCGs with two-sided pc scale jets and dots, one-sided sources.

one-sided jet morphology is the predominant structure and only $22 \%$ of FRI radio galaxies have two-sided jets. This agrees with expectations based on a random orientation for sources with relativistic jets. Based on conclusions for the BCS sample, we suggest that all FRIs outside cool cores have similar parsec-scale properties regardless of their host galaxy classification (BCG or non BCG). One-sided structures in non cool core clusters are produce by to Doppler boosting effects in relativistic, intrinsically symmetric jets.

Two-sided structures can be due to either relativistic jets in the plane of the sky or mildly relativistic jets. For our BCGs, we exclude the first hypothesis because of statistical considerations and after comparison with the BCS results. It is not possible that all BCGs in cool core clusters discussed here with resolved jets are oriented on the plane of sky. We therefore conclude that BCGs in cool core clusters have on the parsec-scale mildly relativistic jets. All resolved BCGs in cool core clusters exhibit twosided jets. This result implies that BCGs in cool core clusters must be due to mildly relativistic jets and they are not a consequence of relativistic jets in the plane of sky. To test this hypothesis, in Fig. 10 we plot the observed total arcsecond radio power at $408 \mathrm{MHz}$ versus the observed arcsecond core radio power at $5 \mathrm{GHz}$ for all the sources in our extended sample. The solid black line is the correlation found by Giovannini et al. (2001) for sources with relativistic jets. According tol Table 3, for UGC 10143, UGC 10187, and NGC 6086, we draw the upper limits of the observed arcsecond core radio power at $5 \mathrm{GHz}$. Despite the low number of sources discussed here we note that the two-sided sources (crosses in the plot) detected in cooling clusters, which are expected to show mildly relativistic jets, do not agree with the correlation found by Giovannini et al. (2001), while the BCG with one-sided jets (dots in the figure) are in good agreement with the general correlation and this confirms the presence of relativistic jets in these BCGs.

\subsection{Mildly relativistic jets}

In BCGs at the center of cooling cores the gas density in the ISM region is expected to be higher (Salomé \& Combes 2003). Studies of the X-ray emission of the hot intra-cluster medium (ICM) have pointed out the high density of this gas in the central regions of many clusters. For example, estimated cooling rates of the order of $10 M_{\odot} / \mathrm{yr}$ and up to $100 M_{\odot} / \mathrm{yr}$ have implied that enormous quantities of material should have accumulated $\left(10^{10}\right.$ to $10^{11} M_{\odot}$ in a fraction of a Hubble time). Because of the dense ISM of BCGs in cool core clusters, we suggest that within BCGs in cool core clusters the jet interaction with the ISM is already relevant on the parsec-scale. We note (see Table 4) that two-sided jets are present only in BCGs at the center of clusters with a central mass accretion rate $>90 M_{\odot} / \mathrm{yr}$.

Rossi et al. (2008) discussed the interaction between relativistic jets and the surrounding ISM. They showed that a jet perturbation grows because of Kelvin-Helmotz instability and produces a strong interaction between the jet and the external medium with a consequent mixing and deceleration. The deceleration becomes more efficient as the density ratio of the ambient medium to the jet increases. Light, relativistic jets are expected in FR I sources, so the above effect can decelerate them from the parsec to the sub-kiloparsec scale as found in many sources (Taylor 1996; Rossi et al. 2008). Since light jet beams imply reduced jet kinetic powers, the model of Rossi et al. (2008) leaves the density contrast as the most likely candidate to account for the discrepancies in the efficiency of the deceleration process. In this scenario, as for sources in non cool core clusters, the jet begin relativistic (and thus one-sided at its base) but a large value of the density ratio can produce a sub-relativistic (and therefore two-sided) heavy jet at a much shorter distance from the central engine compared to "normal" FR I radio galaxies.

\subsubsection{Hydra A and $3 C 84$}

There are two cases in particular, Hydra A and 3C 84 in the cool core clusters A780 and A426, respectively (see Sect. 4), where the above scenario seems to be most evident. Hydra A appears surprisingly symmetric given the observed RM and depolarization asymmetries seen on large scales. Taylor (1996) suggested that the emission from the symmetric parsec-scale jets is more dependent on interactions with the surrounding material than on Doppler boosting. 
In our sample, $3 \mathrm{C} 84$ is the nearest $(z=0.0178)$ and most well studied BCG at high resolution. VLBA images at $86 \mathrm{GHz}$ (Lee et al. 2008) and $43 \mathrm{GHz}$ (Lister 2001) reveal that at a resolution of 0.32 mas $(\sim 0.07 \mathrm{pc})$ the source is one-sided. On larger scales, in VLBA data (Taylor et al. 2006a) 3C 84 shows a two-sided morphology implying that the jet interaction with the dense surrounding medium decelerates the initially relativistic jet on a sub-pc scale.

\section{Conclusions}

BCGs are a unique class of objects. To study their properties on the parsec-scale, we have defined a complete sample selecting all BCGs in nearby Abell clusters $(\mathrm{DC}<3)$ and declination $>0^{\circ}$. We obtained VLBA observations at $5 \mathrm{GHz}$ for these objects without radio data at mas resolution available from literature. We find a different behavior between BCGs in cool core and non cool core clusters. Undetected and point like sources are found in BCGs of both types of clusters. Undetected sources are generally a consequence of low radio activity at the epoch of the observations (radio quiet core), or no radio emission whatsoever (radio quiet source). Point source morphologies may indicate the insufficient sensitivity of our data and/or core dominance effects.

To help us understand their properties, we complemented our sample with other BCGs that have detailed information about the radio emission on the parsec-scale and X-ray properties of the cluster. The extended sample is composed of 34 BCGs: 11 in cool core clusters and 23 in non cool core clusters. A dichotomy is found between the parsec-scale structures of BCGs in cool core and non cool core clusters: all the resolved objects $(56 \%)$ in non cool core clusters show a one-sided jet, whereas in cool core clusters, all the resolved BCGs $(64 \%)$ show a two-sided morphology. Using the BCS sample as a comparison sample, we suggest that one sided structure in non cool core clusters is due to Doppler boosting effects in relativistic, intrinsically symmetric jets. Furthermore, the dominance of two-sided jet structures only in cooling clusters suggests that they have sub-relativistic jet velocities. The different jet properties can be related to different jet origin or the interaction with a different ISM. In BCGs at the center of a cooling core cluster the gas density in the ISM region is expected to be higher. We can therefore assume a strong interaction of the jet at parsec resolution with the environment. However, the large value of the density ratio of the medium to the jet, can produce entrainment leading to a sub-relativistic and heavy jet at a much shorter distance (pc scale). This suggestion is supported by data from the literature on Hydra A and 3C 84, BCGs of two cool core clusters (A780 and A426 respectively). We also found episodic jet activity from the central engine of AGN in a few objects. The recurrent activity of the radio source in cool core clusters is of great interest to the study of AGN feedback in clusters.

More data are necessary to better understand and test the nature of the difference that we note between BCGs in cool and non cool clusters. We would also like to understand the properties of the restarted emission in BCGs. To improve the statistics of our analysis, observations of a larger sample of BCGs in cooling and relaxed clusters with the VLBA are necessary.

Acknowledgements. We thank the staff of NRAO involved in the observations for their assistance. NRAO is a facility of the National Science Foundation, operated under cooperative agreement by Associated Universities, Inc. This research has made use of the NASA/IPAC Extragalactic Data Base (NED), which is operated by the JPL, California Institute of Technology, under contract with the
National Aeronautics and Space Administration. We thanks also an anonymous referee for useful comments which improved this work.

\section{References}

Agudo, I., Krichbaum, T. P., Bach, U., et al. 2007, in "Exploring the Cosmic Frontier: Astrophysical Instruments for the XXI Century", A. P. Lobanov et al. (Eds.), Springer

Allen, S. W., Ettori, S., \& Fabian, A. C. 2001, MNRAS, 324, 877

Asada, K., Nagai, H., Kameno, S., et al. 2009, Contribution in a conference Approaching Micro-arcsecond resolution with VSOP-2: Astrophysics and Technology

Augusto, P., Edge, A. C., \& Chandler, C. J. 2006, MNRAS, 367, 366

Baum, S. A., O’Dea, C. P., Giovannini, G., et al. 1997, ApJ, 483, 178

Becker, R. H., White, R. L., \& Helfand, D. J. 1995, ApJ, 450, 559

Bernardi, M., Nichol, R. C., Sheth, R. K., Miller, C. J., \& Brinkmann, J. 2006, AJ, 131, 1288

Best, P. N., Kaiser, C. R., Heckman, T. M., \& Kauffmann, G. 2006, MNRAS, 368, L67

Best, P. N., von der Linden, A., Kauffmann, G., Heckman, T. M., \& Kaiser, C. R. 2007, MNRAS, 379, 894

Bird, C. M., Davis, D. S., \& Beers, T. C. 1995, AJ, 109, 920

Bîrzan, L., McNamara, B. R., Nulsen, P. E. J., Carilli, C. L., \& Wise, M. W. 2008, ApJ, 686, 859

Blanton, E. L., Sarazin, C. L., McNamara, B. R., \& Wise, M. W. 2001, ApJ, 558, L15

Blanton, E. L., Sarazin, C. L., McNamara, B. R., \& Clarke, T. E. 2004, ApJ, 612, 817

Bliton, M., Rizza, E., Burns, J. O., Owen, F. N., \& Ledlow, M. J. 1998, MNRAS, 301, 609

Böhringer, H., Belsole, E., Kennea, J., et al. 2001, A\&A, 365, L181

Bonamente, M., Lieu, R., Joy, M. K., \& Nevalainen, J. H. 2002, ApJ, 576, 688

Bondi, M., Gregorini, L., Padrielli, L., \& Parma, P. 1993, A\&AS, 101, 431

Brüggen, M., Kaiser, C. R., Churazov, E., \& Enßlin, T. A. 2002, MNRAS, 331, 545

Braine, J., \& Dupraz, C. 1994, A\&A, 283, 407

Burns, J. O. 1990, AJ, 99, 14

Burns, J. O., White, R. A., \& Hough, D. H. 1981, AJ, 86, 1

Burns, J. O., Rhee, G., Owen, F. N., \& Pinkney, J. 1994, ApJ, 423, 94

Celotti, A., \& Fabian, A. C. 1993, MNRAS, 264, 228

Clarke, T. E., Blanton, E. L., Sarazin, C. L., et al. 2009, ApJ, 697, 1481

Chen, Y., Reiprich, T. H., Böhringer, H., Ikebe, Y., \& Zhang, Y.-Y. 2007, A\&A, 466,805

Condon, J. J., Cotton, W. D., Greisen, E. W., et al. 1998, AJ, 115, 1693

Conselice, C. J., Gallagher, J. S., III, \& Wyse, R. F. G. 2001, AJ, 122, 2281

Cowie, L. L., Hu, E. M., Jenkins, E. B., \& York, D. G. 1983, ApJ, 272, 29

Crawford, C. S., Allen, S. W., Ebeling, H., Edge, A. C., \& Fabian, A. C. 1999, MNRAS, 306, 857

Dhawan, V., Kellerman, K. I., \& Romney, J. D. 1998, ApJ, 498, L111

David, L. P., Nulsen, P. E. J., McNamara, B. R., et al. 2001, ApJ, 557, 546

Dickey, J. M. 1997, AJ, 113, 1939

Di Matteo, T., Johnstone, R. M., Allen, S. W., \& Fabian, A. C. 2001, ApJ, 550, L19

Donnelly, R. H., Markevitch, M., Forman, W., et al. 1998, ApJ, 500, 138

Dunn, R. J. H., Fabian, A. C., \& Celotti, A. 2006, MNRAS, 372, 1741

Dunn, R. J. H., Allen, S.W., Taylor, G. B., et al.2010, MNRAS, 404, 180

Ebneter, K., \& Balick, B. 1985, AJ, 90, 183

Edge, A. C. 2001, MNRAS, 328, 762

Eilek, J. A., \& Owen, F. N. 2002, ApJ, 567, 202

Ensslin, T. A., \& Biermann, P. L. 1998, A\&A, 330, 90

Fabian, A. C., Sanders, J. S., Ettori, S., et al. 2000, MNRAS, 318, L65

Fabian, A. C., Celotti, A., Blundell, K. M., Kassim, N. E., \& Perley, R. A. 2002, MNRAS, 331, 369

Fabian, A. C., Sanders, J. S., Allen, S. W., et al. 2003, MNRAS, 344, L43

Fabian, A. C., Reynolds, C. S., Taylor, G. B., \& Dunn, R. J. H. 2005, MNRAS, 363,891

Fabian, A. C., Sanders, J. S., Taylor, G. B., et al. 2006, MNRAS, 366, 417

Fanaroff, B. L., \& Riley, J. M. 1974, MNRAS, 167, 31P

Meier, D. L., Ulrich, M.-H., Fanti, R., Gioia, I., \& Lari, C. 1979, ApJ, 229, 25

Fanti, C., Fanti, R., de Ruiter, H. R., \& Parma, P. 1987, A\&AS, 69, 57

Feretti, L., \& Giovannini, G. 1985, A\&A, 147, L13

Feretti, L., \& Giovannini, G. 1987, A\&A, 182, 15

Feretti, L., \& Giovannini, G. 1988, A\&A, 191, 21

Feretti, L., \& Giovannini, G. 1994, A\&A, 281, 375

Feretti, L., Comoretto, G., Giovannini, G., Venturi, T., \& Wehrle, A. E. 1993, ApJ, 408, 446 
Flin, P. \& Krywult, J. 2006, A\&A, 450, 9

Flin, P., Trevese, D., Cirimele, G., \& Hickson, P. 1995, A\&AS, 110, 313

Fisher, D., Illingworth, G., \& Franx, M. 1995, ApJ, 438, 539

Jones, C., \& Forman, W. 1999, ApJ, 511, 65

Gavazzi, G., \& Jaffe, W. 1987, A\&A, 186, L1

Gentile, G., Rodríguez, C., Taylor, G. B., et al. 2007, ApJ, 659, 225

Ge, J., \& Owen, F. N. 1994, AJ, 108, 1523

Giovannini, G., Feretti, L., Venturi, T., et al. 1994, The Physics of Active Galaxies, 54, 263

Giovannini, G., Cotton, W. D., Feretti, L., Lara, L., \& Venturi, T. 1998, ApJ, 493, 632

Giovannini, G., Cotton, W. D., Feretti, L., Lara, L., \& Venturi, T. 2001, ApJ, 552, 508

Giovannini, G., Taylor, G. B., Feretti, L., et al. 2005, ApJ, 618, 635

Girardi, M., Escalera, E., Fadda, D., et al. 1997, ApJ, 482, 41

Gitti, M., Ferrari, C., Domainko, W., Feretti, L., \& Schindler, S. 2007, A\&A, 470, L25

Gregory, S. A., \& Thompson, L. A. 1984, ApJ, 286, 422

Grillmair, C. J., Faber, S. M., Lauer, T. R., et al. 1994, AJ, 108, 102

Govoni, F., Murgia, M., Markevitch, M., et al. 2009, A\&A, 499, 371

Hudson, D. S., Mittal, R., Reiprich, T. H., et al. 2010, A\&A, 513, A37

Hoessel, J. G., Gunn, J. E., Thuan, T. X. 1980, ApJ, 241, 486

Johnstone, R. M., Allen, S. W., Fabian, A. C., \& Sanders, J. S. 2002, MNRAS, 336, 299

Katayama, H., Hayashida, K., Takahara, F., \& Fujita, Y. 2003, ApJ, 585, 687

Kellerman, K. I., \& Pauliny-Toth, I. 1968, AJ, 73, 874

Krichbaum, T. P., Witzel, A., Graham, D. A., et al. 1992, A\&A, 260, 33

Lara, L., Cotton, W. D., Feretti, L., et al. 1997, ApJ, 474, 179

Lara, L., Feretti, L., Giovannini, G., et al. 1999, ApJ, 513, 197

Lauer, T. R. 1988, ApJ, 325, 49

Ledlow, M. J., \& Owen, F. N. 1995, AJ, 110, 1959

Lester, D. F., Zink, E. C., Doppmann, G. W., et al. 1995, ApJ, 439, 185

Lee, S.-S., Lobanov, A. P., Krichbaum, T. P., et al. 2008, AJ, 136, 159

Lin, Y.-T., \& Mohr, J. J. 2004, ApJ, 617, 879

Lister, M. L. 2001, ApJ, 562, 208

Lister, M. L., Cohen, M. H., Homan, D. C., et al. 2009, AJ, 138, 1874

Liuzzo, E., Taylor, G. B., Giovannini, G., \& Giroletti, M. 2009a, A\&A, 501, 933

Liuzzo, E., Giovannini, G., Giroletti, M., \& Taylor, G. B. 2009b, A\&A, 505, 509

Malumuth, E. M., \& Kirshner, R. P. 1981, ApJ, 251, 508

McNamara, B. R. 1992, BAAS, 24, 1143

McNamara, B. R. 2004, The Riddle of Cooling Flows in Galaxies and Clusters of galaxies, 177

McNamara, B. R., Wise, M., Nulsen, P. E. J., et al. 2000, ApJ, 534, L135

McNamara, B. R., Wise, M. W., Nulsen, P. E. J., et al. 2001, ApJ, 562, L149

McNamara, B. R., Nulsen, P. E. J., Wise, M. W., et al. 2005, Nature, 433, 45

Morganti, R., Killeen, N. E. B., \& Tadhunter, C. N. 1993, MNRAS, 263, 1023

Morris, R. G., \& Fabian, A. C. 2005, MNRAS, 358, 585

Mohr, J. J., Geller, M. J., Fabricant, D. G., et al. 1996, ApJ, 470, 724

Nagai, H., Asada, K., Doi, A., Kameno, S., \& Inoue, M. 2009, Astron. Nachr., 330,161

Nesterov, N. S., Lyuty, V. M., \& Valtaoja, E. 1995, A\&A, 296, 628

O'Donoghue, A. A., Owen, F. N., \& Eilek, J. A. 1990, ApJS, 72, 75
Owen, F. N., Burns, J. O., \& White, R. A. 1984, Clusters and Groups of Galaxies. International Meeting held in Trieste, Italy, September 13-16, ed. F. Mardirossian, G. Giuricin, \& M. Mezzetti (Dordrecht, Holland, Boston, MA, Hingham, MA, USA: D. Reidel Pub. Co.), Sold and distributed in the USA and Canada by Kluwer Academic Publishers, 295

Owen, F. N., \& Ledlow, M. J. 1997, ApJS, 108, 41

Owen, F. N., O’Dea, C. P., Inoue, M., \& Eilek, J. A. 1985, ApJ, 294, L85

Parma, P., de Ruiter, H. R., Fanti, C., \& Fanti, R. 1986, A\&AS, 64, 135

Parma, P., de Ruiter, H. R., \& Cameron, R. A. 1991, AJ, 102, 1960

Pedlar, A., Ghataure, H. S., Davies, R. D., et al. 1990, MNRAS, 246, 477

Peterson, J. R., Ferrigno, C., Kaastra, J. S., et al. 2002, Proc. Symp., New Visions

of the X-ray Universe in the XMM-Newton and Chandra Era, ESA SP 488, ed. F. Jansen [arXiv: astro-ph/0202108]

Rossi, P., Mignone, A., Bodo, G., Massaglia, S., \& Ferrari, A. 2008, A\&A, 488, 795

Ruszkowski, M., Brüggen, M., \& Begelman, M. C. 2004, ApJ, 611, 158

Sakelliou, I., \& Merrifield, M. R. 1999, MNRAS, 305, 417

Salomé, P., \& Combes, F. 2003, A\&A, 412, 657

Salomé, P., \& Combes, F. 2004, A\&A, 415, L1

Silver, C. S., Taylor, G. B., \& Vermeulen, R. C. 1998, ApJ, 502, 229

Schombert, J. M. 1986, ApJS, 60, 603

Scodeggio, M., Solanes, J. M., Giovanelli, R., \& Haynes, M. P. 1995, ApJ, 444, 41

Taylor, G. B. 1996, ApJ, 470, 394

Taylor, G. B., \& Vermeulen, R. C. 1996, ApJ, 457, L69

Taylor, G. B., Perley, R. A., Inoue, M., et al. 1990, BAAS, 22, 802

Taylor, G. B., O'Dea, C. P., Peck, A. B., \& Koekemoer, A. M. 1999, ApJ, 512, L27

Taylor, G. B., Fabian, A. C., \& Allen, S. W. 2002, MNRAS, 334, 769

Taylor, G. B., Gugliucci, N. E., Fabian, A. C., et al. 2006a, MNRAS, 368, 1500

Taylor, G. B., Sanders, J. S., Fabian, A. C., \& Allen, S. W. 2006b, MNRAS, 365, 705

Trussoni, E., Massaglia, S., Ferrari, R., et al. 1997, Mem. Soc. Astron. Ital., 68, 295

Vallee, J. P., Wilson, A. S., \& van der Laan, H. 1979, A\&A, 77, 183

Venturi, T., Readhead, A. C. S., Marr, J. M., \& Backer, D. C. 1993, ApJ, 411, 552

Vermeulen, R. C., Readhead, A. C. S., \& Backer, D. C. 1994, ApJ, 430, L41

Venturi, T., Castaldini, C., Cotton, W. D., et al. 1995, ApJ, 454, 735

Venturi, T., Dallacasa, D., \& Stefanachi, F. 2004, A\&A, 422, 515

Verdoes Kleijn, G. A., Baum, S. A., de Zeeuw, P. T., \& O’Dea, C. P. 1999, AJ, 118,2592

Walker, R. C., Romney, J. D., \& Benson, J. M. 1994, ApJ, 430, L45

Walker, R. C., Kellermann, K. I., Dhawan, V., et al. 1998, Radio Emission from Galactic and Extragalactic Compact Sources, IAU Colloq. 164, 144, 133

Walker, R. C., Dhawan, V., Romney, J. D., Kellermann, K. I., \& Vermeulen, R. C. 2000, ApJ, 530, 233

White, D. A., Jones, C., \& Forman, W. 1997, MNRAS, 292, 419

Wise, M. W., McNamara, B. R., Nulsen, P. E. J., Houck, J. C., \& David, L. P. 2007, ApJ, 659, 1153

Xu, C., Baum, S. A., O’Dea, C. P., Wrobel, J. M., \& Condon, J. J. 2000, AJ, 120, 2950 